\title{
ALGEBRAIC CURVES AND NON RIGID MINIMAL SURFACES IN THE EUCLIDEAN SPACE
}

\author{
Gian Pietro Pirola
}

Using method from algebraic geometry we prove:

Theorem. Let $X$ be a compact connected Riemann surface and $Z$ be a non empty finite subset of $X$. Then there is a complete minimal immersion $F: X-Z \rightarrow \mathbb{R}^{3}$ such that $F(X-Z)$ is non rigid and of finite total Gaussian curvature.

\section{Introduction.}

We study the complete minimal surfaces of bounded total (Gaussian) curvature in the Euclidean space. By the fundamental result of Osserman (see [12], [13]) if $F: Y \rightarrow \mathbb{R}^{3}$ is such a minimal immersion then $Y$ is conformally equivalent to a compact Riemann surface $X$ minus a finite number of points and the Weierstrass data (see 0.19 below) extends meromorphically to $X$. A natural question ([13], page 151) is to give more examples of such surfaces. In [6] it is proved that for any connected compact Riemann surface $X$ there is a finite set of points $Z=\left\{p_{1}, \ldots, p_{n}\right\}$ and a minimal immersion $F: X-Z \rightarrow \mathbb{R}^{3}$ such that $F(X-Z)$ is complete, non rigid and of finite total curvature. Many more examples were found by Yang (cf. [15]) where a bound on the degree of $Z$ is given.

The non rigid minimal surfaces of finite curvature are algebraic geometric objects. In fact a theorem of Calabi implies (cf. [4] and see 0.16, 0.27) that $F$ is the real part of an algebraic morphism $\Phi: X-Z \rightarrow \mathbb{C}^{3}$.

To show our theorem we adopt the spinor viewpoint of [10]. In our opinion it has several advantages. Firstly (see 1.8) the components which correspond to different spin bundles are separated. Then the existence result follows by a Chern class computation. We notice the analogy with the Brill-Noether theory (cf. [1]). This is the easy part of the job.

The duty is to prove that some of those surfaces are indeed immersed. The spinor representation allows to compute the holomorphic tangent space of the moduli variety, say $\mathcal{F}$, representing them. In fact, as is neatly explained in [10], some quadratic equations become linear. We prove, under suitable hypothesis on the polar divisor, that a component of $\mathcal{F}$, say $\mathcal{G}$, has the 
expected dimension and it is generally smooth. By the same argument we show that the subvariety of $\mathcal{G}$ defined by the branched minimal surfaces has codimension one.

The smoothness of the moduli varieties turns out to be equivalent to the surjectivity of certain linear maps obtained by composing a De Rham homomorphism with a suitable multiplication of line bundles. The technical problem is reduced to a single vanishing theorem (see 4.4). This result could be of some independent interest. The question is to know when a space of meromorphic forms, with pole and part of the zero divisor fixed, surjects onto the cohomology of $X-Z$. To perform the various reductions we need the degree of the Gauss map to be quite big. We provide (cf. 5.8) immersions only for curvature approximately bigger than $2 \pi(2 g(n+1)+n(3 n+1)-6)$ if $\operatorname{deg}(Z)=n \geq 8$, which is far to be a good bound. We prefer to avoid further algebraic complications that would occur to obtain better, but not definitive, results (see 5.9).

The paper is organized as follows: In $\S 0$ we give an account of the spinor representation of a minimal surface. We suggest again [10] for an extensive discussion about its geometric and topological meaning. Our effort was to tie the algebraic and the differential geometry involved in a elementary way. In $\S 1$ we fix the notation and discuss the moduli spaces. In $\S 2$ we show the existence of branched minimal surfaces. In $\S 3$ the tangent spaces of the moduli are computed and in $\S 4$ the basic technical problems are solved. The proof of our theorem is completed in $\S 5$.

It is a current opinion that the non-rigid minimal surfaces are not very interesting from any geometric point of view. The author only partially agree with this. They were quite important in R. Bryant's work on Willmore surfaces (cf. [3]), just on genus 0(!). They also provides flat ends examples and non trivial Jacobi fields. In any way, we show that there are not conformal obstructions to give a minimal immersion of an affine algebraic curve in the Euclidean space, which is a natural question.

We would like to thank Francisco Lopez that suggested the problem, encouraged and helped me in many valuable discussions. I would like to thank also Antonio Ros and the Department of Mathematics of Granada for the friendly hospitality.

\section{Spin representation of a minimal surface.}

0.1. Conformal immersions. Let $Y$ be a smooth $\left(C^{\infty}\right)$, connected and oriented surface. Let $F: Y \rightarrow \mathbb{R}^{3}$ be a smooth immersion, that is the differential of $F$ is injective. We assume that $F(Y)$ is not contained in any plane. There is a unique conformal structure on $Y$ such that $F$ is a conformal 
map. Since $Y$ is oriented it is, in a natural way, a Riemann surface. Then there exists a triple of 1.0 differentials $\left(\omega_{1}, \omega_{2}, \omega_{3}\right)$ on $Y$ :

$$
F(q)=\operatorname{Re} \int_{[p, q]}\left(\omega_{1}, \omega_{2}, \omega_{3}\right)+\text { constant }
$$

where $p$ is a fixed point of $X$. We recall that a (1.0) differential $\omega$ on $Y$ has locally the form: $\omega \equiv \phi d z$, where $z=x+i y$ is a holomorphic coordinate and $\phi$ is a smooth complex valued function. The following conditions hold:

$$
\forall \gamma \in H_{1}(Y, \mathbb{Z}), \operatorname{Re} \int_{\gamma} g\left(\omega_{1}, \omega_{2}, \omega_{3}\right)=0 \text {, i.e. } \operatorname{Re}\left(\omega_{i}\right), i=1,2,3 \text {, are exact. }
$$

Conversely if $\left(Y,\left(\omega_{1}, \omega_{2}, \omega_{3}\right)\right)$ satisfies $(0 . a) \ldots(0 . d)$ then $(*)$ defines a conformal immersion:

I) The (0.c) and (0.d) are respectively the local and global compatibility conditions, which imply that $F$ is well defined.

II) (0.b) provides that $F$ is an immersion, $\mathbf{g}$ is the first fundamental form. III) Finally from (0.a) $\mathbf{g}=\lambda\left(d x^{2}+d y^{2}\right): F$ is a conformal map.

(0.2) Definition. The data $\left(Y,\left(\omega_{1}, \omega_{2}, \omega_{3}\right)\right)$ where $Y$ is a Riemann surface and the $\omega_{i}$ are (1.0) differentials on $Y$ is called a Weierstrass data (W-data) if (0.a) and (0.b) are satisfied.

(0.3) Spinors. We recall the classical definition of E. Cartan. Let $q$ : $\mathbb{C}^{3} \rightarrow \mathbb{C}$ be the quadratic form defined by $q\left(x_{1}, x_{2}, x_{3}\right)=\sum_{i=1}^{3} x_{i}^{2}$. Let $I=$ $\left\{\omega \in \mathbb{C}^{3}: q(w)=0\right\}$ be the cone of the $q$-isotropic vectors. Set $S=\mathbb{C}^{2}$ and define $\mathcal{W}: S \rightarrow \mathbb{C}^{3}$ by

$$
\mathcal{W}\left(\zeta_{0}, \zeta_{1}\right)=\left(\zeta_{0}^{2}-\zeta_{1}^{2}, i\left(\zeta_{0}^{2}+\zeta_{1}^{2}\right),-2 \zeta_{0} \zeta_{1}\right)
$$

The image of $\mathcal{W}$ is $I$ and $\mathcal{W}: S-\{(0,0)\} \rightarrow I-\{(0,0,0)\}$ is a 2:1 covering: $\mathcal{W}\left(s_{1}\right)=\mathcal{W}\left(s_{2}\right)$ if and only if $s_{1}= \pm s_{2}$. An element $\mathbf{s}$ of $S$ is a spinor ${ }^{1}$.

(0.5). The Veronese embedding $\mathcal{V}: \mathbb{C P}^{1} \rightarrow \mathbb{C P}^{2}$ is defined by $\mathcal{V}([s])=$ $[\mathcal{W}(s)]$, where [] denote the projective class. The image of $\mathcal{V}$ is the conic

$$
Q=\left\{\left[x_{1}, x_{2}, x_{3}\right] \in \mathbb{C P}^{2}: \sum_{i=1}^{3} x_{i}^{2}=0\right\} .
$$

\footnotetext{
${ }^{1} \mathrm{~A}$ spinor is a sort of "directed" or "polarized" isotropic vector (E. Cartan).
} 
(0.6). Let $\mathcal{O}_{\mathbb{C P}^{n}}(-1)=\left\{(v, \Lambda) \in \mathbb{C}^{n+1} \times \mathbb{C P}^{n}: v \in \Lambda\right\}$ be the tautological line bundle of $\mathbb{C P}^{n}, \mathcal{O}_{\mathbb{C P}^{n}}(1)=\mathcal{O}_{\mathbb{C P}^{n}}(-1)^{-1}$ be its dual and $\mathcal{O}_{\mathbb{C P}^{n}}(n)=$ $\mathcal{O}_{\mathbb{C P}^{n}}(1)^{\otimes n}, n \in \mathbb{Z}, \mathcal{O}=\mathbb{C} \times \mathbb{C P}^{n}$. The coordinates $z_{i}$ of $\mathbb{C}^{n+1}$ give sections of $\mathcal{O}_{\mathbb{C P}^{n}}(1)$. On $\mathbb{C P}^{1}: s \otimes s \rightarrow \mathcal{W}(s)$ induces an identification:

$$
\mathcal{O}_{\mathbb{C P}^{1}}(-2) \equiv \mathcal{V}^{*}\left(\mathcal{O}_{\mathbb{C P}^{2}}(-1)\right)=\left\{(v,[\mathbf{s}]) \in I \times \mathbb{C P}^{1}: v \in \mathcal{V}([\mathbf{s}])\right\}
$$

Dually we have $\mathcal{V}^{*}\left(\mathcal{O}_{\mathbb{C P}^{2}}(1)\right) \equiv \mathcal{O}_{\mathbb{C P}^{1}}(2)$.

(0.8). Set $h: Y \rightarrow \mathbb{C P}^{2}, h(q)=\left[\omega_{1}(q), \omega_{2}(q), \omega_{3}(p)\right]$, which is well defined: By (0.b) the base locus $\left\{p \in Y: \omega_{i}(p)=0, i=1 \leq i \leq 3\right\}$ is empty. By the same reason $\lambda: \mathcal{O}_{Y}^{3} \rightarrow \omega_{Y}, \lambda\left(a_{1}, a_{2}, a_{3}\right)=a_{1} \omega_{1}+a_{2} \omega_{2}+a_{3} \omega_{3}$, is a surjection. Therefore $k: h^{*}\left(\mathcal{O}^{3}\right) \rightarrow \cong \mathcal{O}_{Y}^{3}, k\left(\omega_{i}\right)=z_{i}$, gives an isomorphism: $h^{*}\left(\mathcal{O}_{\mathbb{C P}^{2}}(1)\right) \cong \omega_{Y}$. Since, by $(0 . \mathrm{a}), h(Y) \subset Q$ we may define $f: Y \rightarrow \mathbb{C P}^{1}$ in such a way that $h=\mathcal{V}$.f. Letting $L=f^{*}\left(\mathcal{O}_{\mathbb{C P}^{1}}(1)\right)$ we obtain:

$$
\omega_{Y} \cong f^{*}\left(\mathcal{V}^{*}\left(\mathcal{O}_{\mathbb{C P}^{2}}(1)\right)\right)=f^{*}\left(\mathcal{O}_{\mathbb{C P}^{1}}(2)\right)=f^{*}\left(\mathcal{O}_{\mathbb{C P}^{1}}(1)\right) \otimes f^{*}\left(\mathcal{O}_{\mathbb{C P}^{1}}(1)\right) \cong L^{2}
$$

We have fixed an isomorphism:

$$
\phi: L \otimes L \rightarrow \omega_{Y}
$$

(0.9) Definitions. a) A spin (line) bundle on $Y$ is a couple $(L, \phi)$, where $\phi: L \otimes L \rightarrow \omega_{Y}$ is an isomorphism.

b) Two spin bundles $(L, \phi)$ and $\left(L^{\prime}, \phi^{\prime}\right)$ are equivalent if there is a bundle isomorphism $\alpha: L \rightarrow L^{\prime}$ such that: $\phi^{\prime}(\alpha(x) \otimes \alpha(x))=\phi(x \otimes x)$.

c) A spin structure of $Y$ is an isomorphism class of its spin bundles.

d) The data $(Y, L, \phi, s, t)$ where $(L, \phi)$ is spin bundle on $Y, s, t$ are smooth independent global sections of $L$ will be called a spin data ( $S$-data).

e) A spin data will be said base point free if $\{p \in Y: s(p)=t(p)=0\}=\varnothing$.

We recall that a smooth section $s$ of the spin bundle $L$ has locally the form: $k(z) \zeta, \phi(\zeta \otimes \zeta)=d z$ and $k(z)$ is a smooth function. For sake of notation we will denote the $S$-data $(Y, L, \phi, s, t)$ only by $(Y, L, s, t)$ when confusion should not occur. We often will also put $\phi(a \otimes b)=a b$. Let $s=f^{*}\left(z_{0}\right)$ and $t=f^{*}\left(z_{1}\right)$ be the pull-back of the sections defined in (0.6). We write

$$
\mathcal{W}(s, t)=\left(s^{2}-t^{2}, i\left(s^{2}+t^{2}\right),-2 s t\right)=\left(\omega_{1}, \omega_{2}, \omega_{3}\right),
$$

where $s^{2}=\phi(s \otimes s), t^{2}=\phi(t \otimes t)$ and $s t=\phi(s \otimes t)$.

(0.10). If $(Y, L, s, t)$ is a $S$-data then $\mathcal{W}(s, t)$ satisfies $(0$.a $)$ and $\mathcal{W}(s, t)=$ $\mathcal{W}\left(s^{\prime}, t^{\prime}\right)$ if and only if $s=s^{\prime}$ and $t=t^{\prime}$ or $s=-s^{\prime}$ and $t=-t^{\prime}$. Moreover $\mathcal{W}(s, t)$ is a $W$-data if and only if the $S$-data is base point free. 
(0.11). We stress that the Weierstrass and spin data are equivalent. However from the $S$-data we see directly an important topological and conformal invariant: The spin structure. This is hidden in the $W$-data.

(0.12) Minimal Immersions (see [13]). An immersion $F: Y \rightarrow \mathbb{R}^{3}$ is minimal if the mean curvature of $F(Y)$ vanishes. A conformal map is minimal if and only if it is harmonic. Let $H^{0}\left(Y, \omega_{Y}\right)$ and $H^{0}(Y, L)$ be respectively the space of holomorphic global sections of $\omega_{Y}$ and $L$. Then $F$ is minimal if and only $\omega_{i} \in H^{0}\left(Y, \omega_{Y}\right)$ (locally $\omega_{i} \equiv \phi_{i} d z, \phi_{i}$ holomorphic) and $s, t \in H^{0}(Y, L)$, that is if the spin and $W$-data are holomorphic.

(0.13). Let $(Y, L, s, t)$ be a holomorphic $S$-data. The base locus $B=\{q \in$ $Y: s(q)=t(q)=0\}=\{q \in Y: \mathcal{W}(s, t)(q)=(0,0,0)\}$ is a discrete set. It follows that $F: Y-B \rightarrow \mathbb{R}^{3}$ is an immersion. The points of $B$ are the branch points of $F, F(Y)$ is a minimal surface branched at $B$.

(0.14). The meromorphic function $\frac{t}{s}$ can be identified with $f: Y \rightarrow$ $\mathbb{C P}^{1}$ and with the Gauss map $Y \rightarrow \stackrel{S}{S} \equiv \mathbb{C} \cup\{\infty\} \equiv \mathbb{C P}^{1}$. Usually the Enneper-Weierstrass data of $F$ is $\left(Y, \omega=s^{2}, f\right)$ and $\omega \in H^{1.0}$. The EnneperWeierstrass and the spin representation of the minimal surface (up to a translation) are:

$$
F(q)=\operatorname{Re} \int_{[p, q]}\left(\left(1-f^{2}\right) \omega, i\left(1+f^{2}\right) \omega,-2 f \omega\right)=\operatorname{Re} \int_{[p, q]} \mathcal{W}(s, t) .
$$

(0.15). For a holomorphic spin or $W$-data the condition (0.c) is always satisfied. It is a difficult question to know whenever (0.d) holds: It is the "period" problem. Let $\mathcal{O}_{Y}$ be the sheaf of holomorphic functions on $Y$. The external differential $d=\partial$ defines a De Rham exact sheaf sequence:

$$
0 \rightarrow \mathbb{C} \rightarrow \mathcal{O}_{Y} \stackrel{d}{\rightarrow} \omega_{Y} \rightarrow 0
$$

where $\mathbb{C}$ denotes the constant sheaf. Cohomology induces the exact sequence:

$$
0 \rightarrow H^{0}(Y, \mathbb{C})=\mathbb{C} \rightarrow H^{0}\left(Y, \mathcal{O}_{Y}\right) \stackrel{d}{\rightarrow} H^{0}\left(Y, \omega_{Y}\right) \stackrel{c}{\rightarrow} H^{1}(Y, \mathbb{C}) .
$$

The coboundary $c$ gives the classes of the forms. We may rewrite (0.d) as:

$$
\operatorname{Re}\left(c\left(\omega_{i}\right)\right)=0 \quad i=1,2,3,
$$

here $H^{1}(Y, \mathbb{C}) \equiv H^{1}(Y, \mathbb{R}) \oplus i H^{1}(Y, \mathbb{R})$. If moreover $c\left(\omega_{i}\right)=0, i=1,2,3$, then $\omega_{i}=d F_{i}$, where the $F_{i}$ are holomorphic functions on $Y$. Let $\Phi=$ $\left(F_{1}, F_{2}, F_{3}\right): Y \rightarrow \mathbb{C}^{3}, F=\operatorname{Re} \Phi, \mathrm{F}(\mathrm{Y})$ is the real part of the holomorphic 
curve $\Phi(Y)$. We define the family of the associated surfaces $\Phi_{\theta}: Y \rightarrow \mathbb{R}^{3}$, $\theta \in[0,2 \pi]$, by:

$$
F_{\theta}(q)=\operatorname{Re}\left(e^{i \theta} \Phi\right)
$$

The metric $\mathbf{g}(\theta)$ in (0.b) is independent of $\theta$, that is $F(Y)$ is not rigid. The converse also holds:

(0.16) Calabi's Theorem. A minimal immersion $F: Y \rightarrow \mathbb{R}^{3}$ is not rigid if and only if it is the real part of a holomorphic curve.

Proof. [4] and [11].

(0.17). Let $(Y, L, s, t)$ be the $S$-data of $F$ and $\Pi=\operatorname{span}(s, t)$ be the subspace $H^{0}(Y, L)$ generated by $s$ and $t$. It follows that $F(Y)$ is non rigid if and only for any $\sigma \in \Pi$, then $c\left(\sigma^{2}\right)=0$, i.e. $d(\kappa)=\sigma^{2}$ where $\kappa$ is a holomorphic function. In fact the forms $s^{2}-t^{2}, i\left(s^{2}+t^{2}\right)$ and $-2 s t$ are exact if and only if $s^{2}, t^{2}$ and $(s+t)^{2}$ are exact.

(0.18). Let $K$ be the Gauss curvature of $F(Y)$, we say $F(Y)$ is of bounded curvature if $\int_{Y}|K|<+\infty$. We have the fundamental:

(0.19) Osserman Theorem. Let $F: Y \rightarrow \mathbb{R}^{3}$ be a complete minimal surface, $F(Y)$ has bounded curvature if and only if $Y$ is conformally equivalent to a compact Riemann surface $X$ minus a finite number of points $Z$, $Y=X-Z$, and the forms $\omega_{i}$ extend meromorphically to $Y$.

Proof. [12].

(0.20). Let $F: Y=X-Z \rightarrow \mathbb{R}^{3}$ be given as in (0.19): $X$ is a compact connected Riemann surface and $Z=\left\{p_{1}, \ldots, p_{n}\right\} n>0, p_{i} \in X$. We also denote by $Z=\sum_{i=1}^{n} p_{i}$ its divisor. Let $\left(\omega_{1}, \omega_{2}, \omega_{3}\right)$ be $W$-data of $F$, fix coordinates $z_{j}$ centred in $p_{j} \in Z$. The $\omega_{i}$ extends to $X$ :

$$
\omega_{i}\left(z_{j}\right) \equiv \sum_{k \geq-m_{j}(i)} a_{k, i} z_{j}^{k} d z_{j}
$$

where $a_{m_{j}(i), i} \neq 0$. Set $m_{j}=\max _{i=1,2,3}\left(m_{j}(i)\right)$ and the polar divisor $E=$ $\sum_{j} m_{j} p_{j}$.

(0.21) Remark: $m_{j}>1$. The condition (0.d) implies that the triple residue:

$$
\left(a_{-1,1}, a_{-1,2}, a_{-1,3}\right)=\operatorname{Re} s_{p_{j}}\left(\omega_{1}, \omega_{2}, \omega_{3}\right)=\frac{1}{2 \pi i} \oint\left(\omega_{1}, \omega_{2}, \omega_{3}\right)
$$

is real. If $m_{j} \leq 1$ by (0.a) $\left(a_{-1,1}\right)^{2}+\left(a_{-1,2}\right)^{2}+\left(a_{-1,3}\right)^{2}=0$, that is $a_{-1, i}=$ $0: m_{j}=0$. This is impossible. If $F$ extends on $p_{j}$ and $(Y, g)$ is not complete. 
(0.22). Let $\omega_{X}(E)$ be the sheaf of the meromorphic forms having polar divisor contained in $E$. Recall that $\omega_{X}(E)$ can be identified with the sheaf of the holomorphic sections of a line bundle on $X$ (see [1] or [7]) which is usually still denoted by $\omega_{X}(E)$. We may consider $\omega_{i} \in H^{0}\left(X, \omega_{X}(E)\right)$. The (0.8) extends and by abuse of language we write $h: X \rightarrow \mathbb{C P}^{2}, h(X)=Q$. Repeating the square root extraction: $h=\mathcal{V} f, f: X \rightarrow \mathbb{C P}^{1}$ is the extended Gauss map. Set $M=f^{*}\left(\mathcal{O}_{\mathbb{C P}^{1}}(1)\right): M^{2}=\omega_{X}(E)$. We find two sections of $M$, called again $s$ and $t$, such that $\mathcal{W}(s, t)=\left(\omega_{1}, \omega_{2}, \omega_{3}\right)$. Note that the restriction of $M$ to $Y$ is $L$.

(0.23). To obtain a canonical extension of $L$ to $X$ it is convenient to divide $Z$ in the two pieces: $Z^{\prime}=\left\{p \in Z: m_{i}\right.$ odd $\}$ and $Z^{\prime \prime}=\left\{p \in Z: m_{i}\right.$ even $\}$. Then $E=2 D+Z^{\prime}, D=\sum n_{i} p_{i}, 2 n_{i}=m_{i}$ if $p_{i} \in Z^{\prime \prime}$ and $2 n_{i}+1=m_{i}$ if $p_{i} \in Z^{\prime}$. Set $\mathbf{L}=M \otimes \mathcal{O}_{X}(-D), \mathbf{L}^{2}=\omega_{X}\left(Z^{\prime}\right), s$ and $t$ are sections of $\mathbf{L}(D)=M: \mathbf{L}_{\mid Y}=L$.

We remark that $(L, \phi)$ determines completely $\mathbf{L}$. To see this let $D_{j}$ be an open disk centred on $p_{j}$ and set $D^{*}=D_{j}-\left\{p_{j}\right\}$. By restriction $(L, \phi)$ defines a spin structure on $D^{*}$. There are exactly two spin structures on $D^{*}$, $L=\mathcal{O}_{D^{*}}$ :

1) $\phi(\kappa \otimes \kappa)=\kappa^{2} d z \Leftrightarrow \omega_{D} \cong \mathbf{L}_{\mid D^{*}}^{2} \Leftrightarrow p_{j} \in Z^{\prime \prime}$.

2) $\phi(\kappa \otimes \kappa)=\kappa^{2} \frac{d z}{z} \Leftrightarrow \omega_{D}\left(p_{j}\right)_{\mid D^{*}} \cong \mathbf{L}_{\mid D^{*}}^{2} \Leftrightarrow p_{j} \in Z^{\prime}$.

Conversely, $\mathbf{L}$ determines $(L, \phi)$. In fact the number of holomorphic line bundles $\mathbf{L}$, such that $\omega_{X} \subset \mathbf{L}^{2} \subset \omega_{X}(Z)$ is $2^{2 g+n-1}$, which is the number of the spin structures on $Y$ (cf. [10]).

(0.24). By abuse of notation we will write $L$ instead of $\mathbf{L}$. From (0.19) the (extended) data of bounded curvature complete minimal surfaces are:

Weierstrass: $\left(X,\left(\omega_{1}, \omega_{2}, \omega_{3}\right)\right), \omega_{i} \in H^{0}\left(X, \omega_{X}(E)\right) E=\sum_{i} m_{i} p_{i}, m_{i}>1$.

Spin: $(X, L(D), s, t), s, t \in H^{0}(X, L(D)), D=\sum_{i} n_{i} p_{i}, n_{i}>0\left(L^{2}(2 D)=\right.$ $\left.\omega_{X}(E)\right)$.

(0.25). Recall that $F: Y=X-Z \rightarrow \mathbb{R}^{3}$ has embedded ends if for any $j$ there is a disk $D_{j}$ centred at $p_{j}$ such that $F\left(D_{j}-p_{j}\right)$ is embedded. In this case (cf. [9]) $m_{j}=2, D=2 Z$ and $L^{2}=\omega_{X}: L$ is a spin bundle of $X$. If $F$ is a global embedding then (cf. [14] and [2]) $L$ is an even spin bundle: $\operatorname{dim}\left(H^{0}(X, L)\right)=2 n \in \mathbb{Z}$.

As a straightforward application we obtain:

(0.26) Jorge-Meeks Formula (cf. [9]): Let $f$ be the extended Gauss map of $F$. Then $\operatorname{deg}(f)=\operatorname{deg}(L(D))=g-1+\frac{\operatorname{deg}\left(Z^{\prime}\right)}{2}+\operatorname{deg}(D)$. If the ends of $F(Y)$ are embedded: $\operatorname{deg}(f)=g-1+\operatorname{deg}(Z)=-\frac{1}{2} \chi(Y)=-\frac{1}{4 \pi} \int_{Y} K$.

(0.27). The differential $(0.15)$ extends to $d: \mathcal{O}_{X}(E-Z) \rightarrow \omega_{X}(E) \quad(E=$ 
$\left.2 D+Z^{\prime}\right)$. This provides an exact sequence in cohomology:

$$
0 \rightarrow \mathbb{C} \rightarrow H^{0}\left(X, \mathcal{O}_{X}(E-Z)\right) \stackrel{d}{\rightarrow} H^{0}\left(X, \omega_{X}(E)\right) \stackrel{c}{\rightarrow} H^{1}(X-Z, \mathbb{C}) .
$$

The condition (0.d)' gives $\operatorname{Re}\left(c\left(\omega_{i}\right)\right)=0 i=1,2,3$. We recall that $F$ has flat ends if $\operatorname{Re} s_{p_{j}}\left(\omega_{1}, \omega_{2}, \omega_{3}\right)=0$ for any $j$. This means that $c\left(\omega_{i}\right)$ belongs to $i^{*}\left(H^{1}(X, \mathbb{C})\right)$ where $i: X-Z \rightarrow X$ is the inclusion.

In the non-rigid minimal case (of bounded curvature) (see 0.15), $\left(\omega_{1}, \omega_{2}\right.$, $\left.\omega_{3}\right)=\left(d F_{1}, d F_{2}, d F_{3}\right)$, the $F_{i}$ are rational functions with poles in $E-Z$. In particular $F=\operatorname{Re} \Phi$ where

$$
\Phi=\left(F_{1}, F_{2}, F_{3}\right): X-Z \rightarrow \mathbb{C}^{3}
$$

is an algebraic morphism (see [8] Ch. 1). We note that a non rigid minimal surface has flat ends. Moreover, by $(0.17), F$ is non rigid if and only for any $\sigma \in \operatorname{span}(s, t) \subset H^{0}(X, L(D))$ there is a rational function $\kappa$ such that $d \kappa=\sigma^{2}$. The ramification divisor of $\kappa$ is twice the divisor of $\sigma$. These minimal surfaces can therefore be studied by means of rational functions having even ramification divisors. This will be a topic of this paper.

\section{Notations and basic definition.}

The following notation will be fixed all over the paper.

- Let $X$ be a compact connected Riemann surface of genus $g$.

- Let $Z=\left\{p_{1}, \ldots, p_{n}\right\} n>0, p_{i} \in X$. We also denote by $Z=\sum_{i=1}^{n} p_{i}$ its divisor.

- Fix $k, 0 \leq 2 k \leq n$, and $Z^{\prime}=\sum_{i=1}^{n} p_{i}\left(Z^{\prime}=0\right.$ if $\left.k=0\right)$ and write $Z=Z^{\prime}+Z^{\prime \prime}$.

- Let $L$ be a holomorphic bundle of $X$ such that $L^{2}=\omega_{X}\left(Z^{\prime}\right), L$ defines a spin structure of $X-Z$ (see [10] and 0.23 above).

- Let $D=\sum_{i} n_{i} p_{i}, n_{i}>0$, be a divisor supported on $Z$, set $d=$ $\operatorname{deg}(D)=\sum_{i=1}^{n} n_{i}$.

- For any divisor, say $E=\sum_{i} k_{i} q_{i}$ where the $q_{i}$ are distinct and $k_{i} \neq 0$, we will denote by $[E]=\sum_{i} q_{i}$ its support divisor, in particular $[D]=Z$.

- If $A=\Sigma_{p \in X} a_{p} p$ and $B=\Sigma_{p \in X} b_{p} p$ are effective divisors, i.e. $a_{p} \geq$ $0, b_{p} \geq 0$ and $\neq 0$ on a finite set of points, we define $A \cap B=$ $\Sigma_{p \in X} \min \left(a_{p}, b_{p}\right) p$. Note that $[A \cap B]=[A] \cap[B]$ and $[A+B]=$ $[A]+[B]-[A \cap B]$.

- We consider the line bundles $\mathcal{O}_{X}\left(2 D-Z^{\prime \prime}\right), L(D)=L \otimes \mathcal{O}_{X}(D)$ and $L(D)^{2}=\omega_{X}\left(2 D+Z^{\prime}\right)$. We let $\mathcal{O}_{\mathbb{C P}^{n}}(1)$ be the tautological line bundle of the complex projective space $\mathbb{C P}^{n}$. 
- Set $H^{0}\left(X, \mathcal{O}_{X}\left(2 D-Z^{\prime \prime}\right)\right)=R, H^{0}(X, L(D))=V, W=H^{0}\left(X, \omega_{X}(2 D+\right.$ $\left.Z^{\prime}\right)$ ) and if $F$ is an effective divisor $V(-F)=H^{0}(X, L(D-F))$.

- $\quad R$ is a space of rational functions (poles at $2 D-Z^{\prime \prime}$ ), $W$ of differentials (poles at $2 D+Z^{\prime}$ ) and $V$ of $L$ "half-differentials" (poles at $D$ ) of $X$. Set $H=H^{1}(X-Z, \mathbb{C}), \operatorname{dim}(H)=2 g+n-1$.

- The exterior differential (see 0.15) defines a mapping: $d: R \rightarrow W$. If $R \neq 0$ (e.g. $2 d+2 k-n>g-1$ ) it gives rise to a exact sequence:

$$
0 \rightarrow H^{0}(X-Z, \mathbb{C})=\mathbb{C} \rightarrow R \stackrel{d}{\rightarrow} W \stackrel{c}{\rightarrow} H .
$$

- Define the maps: $m: \mathrm{Sym}^{2} V \rightarrow W m\left(s_{1} \otimes s_{2}\right)=s_{1} \cdot s_{2}, \theta: \mathrm{Sym}^{2} V \rightarrow$ $H \theta\left(s_{1} \otimes s_{2}\right)=c\left(s_{1} \cdot s_{2}\right)$ and $\theta^{\prime}: V \rightarrow H$ by $\theta^{\prime}(s)=\theta(s \otimes s)=c\left(s_{2}\right)$.

After settled the basic notation we start with some new definitions followed by a few comments.

(1.1) Definition. An element $s$ of $V$ will be called half-exact if $c\left(s^{2}\right)=0$, i.e. $d(f)=s^{2}$ with $f \in R$. Set $\mathcal{P}=\{s \in V: s$ is half-exact $\}$ and $\mathbb{P}(V)$ the projective space of $V$ and $\mathcal{H}=\{[s] \in \mathbb{P}(V): s \in \mathcal{P}\}, \mathcal{P}$ is the affine cone of $\mathcal{H}$.

(1.2). Let $N$ be a subspace of $V$ and $\mathbb{P}(N) \subset \mathbb{P}(V)$ the associated projective space. Set $\mathcal{P}(N)=N \cap \mathcal{P}, \mathcal{H}(N)=\mathbb{P}(N) \cap \mathcal{H}, \mathbb{P}(V(-F))=\mathbb{P}(F)$ and $\mathcal{P}(V(-F))=\mathcal{P}(F)$ when $N=V(-F)$ and $F$ is an effective divisor of $X$.

(1.3) Definition. A subspace $\Pi$ of $V$ will be called a minimal plane if $\operatorname{dim}(\Pi)=2$ and all its elements are half-exact. The minimal planes corresponds to the lines of $\mathbb{P}(V)$ contained in $\mathcal{H}$. A plane $\Pi=\operatorname{span}(s, t)$ will be called base point free if $\{p \in X, s(p)=t(p)=0\} \equiv \varnothing$.

We remark that if $\Pi=\operatorname{span}(s, t)$ is a base point free minimal plane then $(X, L, s, t)$ is base point free $S$-data $(0.9 \mathrm{e})$. Hence by $(0.17)$ we have:

$(X, L, s, t)$ is $S$-data of a non rigid minimal immersion $\Leftrightarrow$ $\Pi=\operatorname{span}(s, t)$ is a base point free minimal plane.

(1.4). Let $G(2, V)$ be the Grassmannian of 2 dimensional space and $\mathcal{M}=$ $\{\Pi \in G(2, V): \Pi$ is a minimal $\}$. We also define the two frame space of $\mathcal{M}$ :

$$
\mathcal{F}=\left\{\left(s_{1}, s_{2}\right) \in V \times V: \operatorname{span}\left(s_{1}, s_{2}\right) \in \mathcal{M}\right\} .
$$

Note that $\mathcal{F} \subset \mathcal{P} \times \mathcal{P}$. We have $\mathcal{F} \neq \varnothing \Leftrightarrow \mathcal{M} \neq \varnothing$ and if $\mathcal{M} \neq \varnothing \Rightarrow$ $\operatorname{dim}(\mathcal{F})=\operatorname{dim}(\mathcal{M})+4$.

(1.5). As before if $N \subset V$ is a subspace, we let $\mathcal{F}(N)=(N \times N) \cap \mathcal{F}$ and $\mathcal{M}(N)=\mathcal{M} \cap G(2, N)$. The inclusion $G(2, N) \subset G(2, V)$ is the natural 
one. If $N=V(-F)$ set $\mathcal{M}(N)=\mathcal{M}(F)$ and $\mathcal{F}(N)=\mathcal{F}(F)$. Note that $\mathcal{M}^{\prime}=\mathcal{M}-\cup_{p \in X} \mathcal{M}(p)$ and $\mathcal{F}^{\prime}=\mathcal{F}-\cup_{p \in X} \mathcal{F}(p)$ give the base point free loci. For $s \in \mathcal{F}$ we set $\mathcal{F}(s)=\left\{\left(s_{1}, s_{2}\right) \in \mathcal{F}: s_{1}=s\right\}$ and $\mathcal{M}(s)=$ $\{\Pi \in \mathcal{M}: s \in \Pi\}$. If $\lambda_{1}: \mathcal{F} \rightarrow V$ is the projection $\lambda_{1}\left(s_{1}, s_{2}\right)=s_{1}$ then $\lambda_{1}^{-1}(s)=\mathcal{F}(s)$ and $\operatorname{dim} \mathcal{F}(s)=\operatorname{dim} \mathcal{M}(s)+2$. Set $\mathcal{M}(s, N)=\mathcal{M}(s) \cap \mathcal{M}(N)$, $\mathcal{M}(s, F)=\mathcal{M}(s) \cap \mathcal{M}(F)$ etc..

(1.6). The two frame space of $V$

$$
s=\left\{\left(s_{1}, s_{2}\right) \in V \times V: \operatorname{dim}\left(\operatorname{span}\left(s_{1}, s_{2}\right)\right)=2\right\}
$$

is by definition the locus of the holomorphic $S$-data (see 0.13 and 0.27 ). Set $\mathcal{S}(p)=\left\{\left(s_{1}, s_{2}\right) \in \mathcal{S}: s_{1}(p)=s_{2}(p)=0\right\}$ and $\mathcal{S}^{\prime}=\mathcal{S}-\cup_{p \in X} \mathcal{S}(p)$. The sublocus (1.4), $\mathcal{F}$, of $\mathcal{S}$ corresponds to the $S$-data of non rigid (branched) minimal surfaces. The locus of the branched $S$-data is $\mathcal{G}=\cup_{p \in X} \mathcal{F}(p)$.

(1.7) Moduli. We denote by $\mathcal{Q}\left(\mathcal{Q}_{\text {flat }}\right)$ respectively the locus of the spin data of minimal surfaces (with flat ends):

$$
\begin{aligned}
\mathcal{Q} & =\left\{\left(s_{1}, s_{2}\right) \in \mathcal{S}: \operatorname{Re} \int_{\gamma} \mathcal{W}\left(s_{1}, s_{2}\right)=0, \gamma \in H^{1}(Y, \mathbb{Z})\right\} \\
\mathcal{Q}_{\text {flat }} & =\left\{\left(s_{1}, s_{2}\right) \in \mathcal{Q}: \operatorname{Re} s_{p_{j}} \mathcal{W}\left(s_{1}, s_{2}\right)=0, p_{j} \in Z\right\} .
\end{aligned}
$$

We have a filtration:

$$
\mathcal{F} \subset \mathcal{Q}_{\text {flat }} \subset \mathcal{Q} \subset \mathcal{S}
$$

The immersions correspond to $\mathcal{Q}^{\prime}=\mathcal{Q} \cap \mathcal{S}^{\prime}$. It could be that $\mathcal{F}=\varnothing$ and $\mathcal{Q} \neq 0$ as the cases of embedded minimal surfaces show.

(1.8). Clearly $\mathcal{F}$ is a moduli space of the non rigid spin minimal surfaces with bounded curvature. We discuss the moduli of the minimal immersions of $X-Z$ by using spinors. Let $\mathcal{I}=\left(k_{1}, \ldots, k_{n}\right), k_{i} \in \mathbb{Z}, k_{i}>0$ be a $n$-ple, set $D_{\mathcal{I}}=\sum_{i}$. Fix a line bundle $L$ on $X$ such that $L^{2}=\omega_{X}\left(Z^{\prime}\right)$ where $Z^{\prime} \subset Z$. Now $L$ (see 0.23 ) defines a spin structure on $X-Z$. Set $V_{\mathcal{I}, L}=H^{0}\left(X, L\left(D_{\mathcal{I}}\right)\right.$ ), $\mathcal{S}_{\mathcal{I}, L}=\mathcal{S}=\left\{\left(s_{1}, s_{2}\right) \in V_{\mathcal{I}, L} \times V_{\mathcal{I}, L}: \operatorname{dim}\left(\operatorname{span}\left(s_{1}, s_{2}\right)\right)=2\right\}$ and accordingly:

$$
\begin{aligned}
& \mathcal{F}_{\mathcal{I}, L} \subset \mathcal{Q}_{\text {flat }, \mathcal{I}, L} \subset \mathcal{Q}_{\mathcal{I}, L} \subset \mathcal{S}_{\mathcal{I}, L} \\
& \mathcal{F}_{\mathcal{I}, L}^{\prime} \subset \mathcal{Q}_{\text {flat }, \mathcal{I}, L}^{\prime} \subset \mathcal{Q}_{\mathcal{I}, L}^{\prime} \subset \mathcal{S}_{\mathcal{I}, L}^{\prime} .
\end{aligned}
$$

If $\mathcal{I}=\left(k_{1}, \ldots, k_{n}\right)$ and $\mathcal{K}=\left(k_{1}^{\prime}, \ldots, k_{n}^{\prime}\right)$ we say that $\mathcal{I} \geq \mathcal{K}$ if $k_{i} \geq k_{i}^{\prime}$ for all $i$. If $\mathcal{I} \geq \mathcal{K}$ there is a natural inclusion $V_{\mathcal{K}, L} \subset V_{\mathcal{I}, L}$ and hence:

$$
\mathcal{F}_{\mathcal{K}, L} \subset \mathcal{F}_{\mathcal{I}, L} ; \mathcal{Q}_{\mathcal{K}, L} \subset \mathcal{Q}_{\mathcal{I}, L} ; \mathcal{S}_{\mathcal{K}, L} \subset \mathcal{S}_{\mathcal{I}, L}
$$

We may consider

$$
\cup_{\mathcal{I}, L} \mathcal{F}_{\mathcal{I}, L} \subset \cup_{\mathcal{I}, L} \mathcal{Q}_{\mathcal{I}, L} \subset \cup_{\mathcal{I}, L} \mathcal{S}_{\mathcal{I}, L} ; \cup_{\mathcal{I}, L} \mathcal{F}_{\mathcal{I}, L}^{\prime} \subset \cup_{\mathcal{I}, L} \mathcal{Q}_{\mathcal{I}, L}^{\prime}
$$


with the induced topology: A set $C \subset \cup_{\mathcal{I}, L} \mathcal{S}_{\mathcal{I}, L}$ is closed if $C \cap \mathcal{S}_{\mathcal{I}, L}$ is closed for all $\mathcal{I}$ and $L$. Note that if $\mathcal{I} \geq \mathcal{K}$ then $\mathcal{S}_{\mathcal{K}, L}$ is closed in $\mathcal{S}_{\mathcal{I}, L}$. Now

$$
\mathbf{Q}(\mathbf{X}, \mathbf{Z})=\cup_{\mathcal{I}, L} \mathcal{Q}_{\mathcal{I}, L}^{\prime}
$$

is the space of the spin data of the minimal immersions of $X-Z$ in the euclidean space. If we fix $L$ and let $\mathbf{Q}(\mathbf{X}, \mathbf{Z}, \mathbf{L})=\cup_{\mathcal{I}} \mathcal{Q}_{\mathcal{I}, L}^{\prime}$ we have:

$$
\mathbf{Q}(\mathbf{X}, \mathbf{Z})=\cup_{L} \mathbf{Q}(\mathbf{X}, \mathbf{Z}, \mathbf{L}) .
$$

We note that $\mathbf{Q}(\mathbf{X}, \mathbf{Z}, \mathbf{L})$ is an open and closed subset of $\mathbf{Q}(\mathbf{X}, \mathbf{Z})$. Let $j$ : $\mathbf{Q}(\mathbf{X}, \mathbf{Z}) \rightarrow \mathbf{Q}(\mathbf{X}, \mathbf{Z})$ be the involution $j(s, t)=(-s,-t)$. The points of the quotient $\mathbf{M}(\mathbf{X}, \mathbf{Z})=\mathbf{Q}(\mathbf{X}, \mathbf{Z}) / \mathbf{j}$ corresponds to the $W$-data having poles at $Z$.

(1.9). We call $\mathbf{M}(\mathbf{X}, \mathbf{Z})$ the moduli space of the complete minimal immersions of bounded curvature of $X-Z$ in $\mathbb{R}^{3}$. We will show in (5.8) that $\mathbf{M}(\mathbf{X}, \mathbf{Z})$ is not empty and more precisely that $\mathcal{F}_{\left(k_{1}, \ldots, k_{n}\right), L}^{\prime} \neq \varnothing$ if $k_{i} \gg 0$ for all $i$. Letting $\mathbf{M}(\mathbf{X}, \mathbf{Z}, \mathbf{L})=\mathbf{Q}(\mathbf{X}, \mathbf{Z}, \mathbf{L}) / \mathbf{j}$ we have $\mathbf{M}(\mathbf{X}, \mathbf{Z})=\cup_{L} \mathbf{Q}(\mathbf{X}, \mathbf{Z}, \mathbf{L})$, where the $\mathbf{M}(\mathbf{X}, \mathbf{Z}, \mathbf{L})$ are not empty open and closed sub-sets.

(1.10) Warning. We note that $\mathbf{M}(\mathbf{X}, \mathbf{Z})$ is not, in general, a good moduli space of the immersed surfaces $F(X-Z)$. This is not due only to the effect of the translations, which could be ignored. If $\mathbf{a}$ is an automorphism of $X$ such that $\mathbf{a}(Z)=Z$, then the $W$-data $\left(\mathbf{a}^{*}\left(\omega_{1}\right), \mathbf{a}^{*}\left(\omega_{2}\right), \mathbf{a}^{*}\left(\omega_{3}\right)\right)$ and $\left(\omega_{1}, \omega_{2}, \omega_{3}\right)$ give the same image. To get the actual moduli space here one should take the quotient by the automorphisms action.

(1.11). The set of all the minimal complete immersions of bounded curvature is $\cup_{X, Z} \mathbf{M}(\mathbf{X}, \mathbf{Z})$. To define however a good topology and possibly other structures, one needs to consider holomorphic $S$-data (or $W$-data) on families of Riemann surfaces. We do not perform here it furthermore.

\section{Existence Results.}

We keep the previous notations. We have introduced the vector spaces $V=$ $H^{0}(X, L(D)), W=H^{0}\left(X, \omega_{X}\left(2 D+Z^{\prime}\right)\right)$ and $H=H^{1}(X-Z, C)$. Then for any subspace $N$ of $V$ we have defined the loci $\mathcal{H}(N), \mathcal{M}(N), \mathcal{F}(N), \mathcal{M}(s, N)$, etc. (cf. 1.1, ., 1.5). In the sequel we will always have $\operatorname{dim}(V)>g$, in particular $L(D)$ will be non-special and hence $\operatorname{dim}(V)=d+k$. We recall that $d=\operatorname{deg}(D), n=\operatorname{deg}(Z)$ and $[D]=Z$. We will use the convention that the dimension of the empty set is $-\infty$, hence $\operatorname{dim}(S) \geq 0$ implies $S \neq \varnothing$.

Proposition 2.1. Let $N$ be a subspace of $V$, $\operatorname{dim}(N)=e$. Assume $e \geq 2 g-n$ then

i) $\operatorname{dim}(\mathcal{H}(N)) \geq e-2 g-n$; 
ii) $\operatorname{dim}(\mathcal{M}(N)) \geq 2 e-3(2 g+n)-1$;

iii) If $[s] \in \mathcal{H}(N)$, then $\operatorname{dim}(\mathcal{M}(s, N)) \geq e-(4 g+2 n)$.

Proof. Set $\operatorname{dim}(H)=2 g+n-1=f$. We will describe our loci as zero sections of vector bundles. First at all we restrict the map $\theta$ of $\S 1$ to $\operatorname{Sym}^{2} N$. This defines $\theta(N): \operatorname{Sym}^{2} N \rightarrow H$. By abuse of notations we will write $\theta=\theta(N)$ :

$$
\theta(s \otimes t)=c(s t)=\text { De Rham class of }\{s t\} .
$$

i) Set $\mathcal{O}(k)=\mathcal{O}_{\mathbb{P}(N)}(k)$ and consider the tautological sequence on $\mathbb{P}(N)$ :

$$
0 \rightarrow \mathcal{O}(-1) \rightarrow N \rightarrow Q \rightarrow 0
$$

where $Q$ is the quotient bundle. Taking symmetric product we get a map $\operatorname{Sym}^{2}(\mathcal{O}(-1)) \equiv \mathcal{O}(-2) \rightarrow \operatorname{Sym}^{2} N$ and, by composing with $\theta, \mu^{\prime}: \mathcal{O}(-2) \rightarrow$ $H$. This gives a section $\mu$ of $H \otimes \mathcal{O}(2)$ and $\mathcal{H}(N)$ is the zero locus of $\mu$ :

$$
\mathcal{H}(N)=\left\{x \in \mathbb{P}(N): \mu(x)=0=\mu^{\prime}(x)\right\} .
$$

Let $\zeta=c_{1}(\mathcal{O}(1))$ be the Chern class of $\mathcal{O}(1)$, that is the hyperplane section. Since $H$ is a trivial bundle the top Chern class of $H \otimes \mathcal{O}(2) \equiv \mathcal{O}(2)^{f}$ is:

$(2.2)$.

$$
c_{f}(H \otimes \mathcal{O}(2))=2^{f} \zeta^{f} .
$$

Assume $\operatorname{dim}(\mathbb{P}(N))=e-1 \geq f$, then $\mathcal{H}(N)$ is not empty and the dimension of any component is bigger than $e-1-f$.

ii) The proof is similar to the previous one. Consider on $G(2, N)$ the tautological sequence of vector bundles:

$$
0 \rightarrow S \rightarrow N \rightarrow \mathcal{Q} \rightarrow 0
$$

The composition $\operatorname{Sym}^{2} S \rightarrow \operatorname{Sym}^{2} N \rightarrow H$ defines $\lambda: \operatorname{Sym}^{2} S \rightarrow H$. We have (2.3).

$$
\mathcal{M}(N)=\{x \in G(2, N): \lambda(x)=0\} .
$$

Let $S^{*}$ be the dual of $S$. We identify $\lambda$ as a section of $H \otimes \operatorname{Sym}^{2} S^{*}$. The result follows by computing the top Chern class:

$$
c_{3 f}\left(H \otimes \operatorname{Sym}^{2} S^{*}\right)=c_{3}\left(\operatorname{Sym}^{2} S^{*}\right)^{f}=4^{f} c_{2}\left(S^{*}\right)^{f}\left(c_{1}\left(S^{*}\right)\right)^{f} \neq 0 .
$$

In fact $c_{1}\left(S^{*}\right)$ is ample and $c_{2}\left(S^{*}\right)^{f} \neq 0$ if $f \leq e: c_{2}\left(S^{*}\right)^{e}=1$ (see [7] Ch. 3 $\S 3)$.

iii) Fix a half-exact element $s$ of $N, s \neq 0$. Set $\mathbb{P}=\{\Pi \in G(2, N): s \in \mathbb{P}\}$, $\mathbb{P} \cap \mathcal{M}=\mathcal{M}(s, N)$. Now $\mathbb{P}$ is isomorphic to the projective space of the 
quotient of $N$ by the space generated by $s$. Let $\mathcal{O}_{\mathbb{P}}(-1)$ be the tautological bundle of $\mathbb{P}$ and $S_{\mathbb{P}}=S \otimes \mathcal{O}_{\mathbb{P}}$ the restriction of $S$ to $\mathbb{P}$. Define the splitting exact sequence:

$$
0 \rightarrow \mathcal{O}_{\mathbb{P}} \stackrel{s}{\rightarrow} S_{\mathbb{P}} \rightarrow \mathcal{O}_{\mathbb{P}}(-1) \rightarrow 0
$$

where $\mathcal{O}_{\mathbb{P}} \rightarrow S_{\mathbb{P}}$ is induced by $s: \operatorname{Sym}^{2} S_{\mathbb{P}}=\mathcal{O}_{\mathbb{P}} \oplus \mathcal{O}_{\mathbb{P}}(-1) \oplus \mathcal{O}_{\mathbb{P}}(-2)$. Denote still by $H$ its restriction to $\mathbb{P}$. The map $\operatorname{Sym}^{2} S \rightarrow H$ defines a section $\sigma$ of $H \otimes \mathcal{O}(1) \oplus H \otimes \mathcal{O}(2) \equiv \mathcal{O}(1)^{f} \oplus \mathcal{O}(2)^{f}$ such that $\mathcal{M}(s, N)=\{\Pi \in \mathbb{P}:$ $\sigma(\Pi)=0\}=Z(\sigma)$. Since $c_{2 f}(H \otimes \mathcal{O}(1) \oplus H \otimes \mathcal{O}(2))=2^{f} \zeta^{2 f}$ it follows that $\operatorname{dim}(\mathcal{M}(s, N)) \geq e-2-2 f$.

Corollary 2.4. i) $\operatorname{dim}(\mathcal{H}(F)) \geq d+k-2 g-n-\operatorname{deg}(F), \operatorname{dim}(\mathcal{H}) \geq$ $d+k-2 g-n$; ii) $\operatorname{dim}(\mathcal{M}(F)) \geq 2(d+k-\operatorname{deg}(F))-3(2 g+n)-1$; iii) If $(s) \in \mathcal{H}(F)$, then $\operatorname{dim}(\mathcal{M}(s, F)) \geq d+k-4 g-2 n-\operatorname{deg}(F)$.

In particular we get:

Proposition 2.5. If $2(d-k)>3(2 g+n)$ then there are (possibly) branched minimal surfaces with polar divisor at $Z$, moreover $\operatorname{dim}(\mathcal{M}) \geq 2(d+k)-$ $3(2 g+n)-1$.

(2.6) Algebraic structure. All previous spaces are zero sections of vector bundles (cf. 2.2 and 2.3) over quasi-projective varieties. We discuss the induced scheme structure (for details see [8] Ch. 1 and 2).

We recall that a Zariski closed set of $\mathbb{C P}^{n}$ is the zero set of a finite number of homogeneous polynomials. Let $\mathcal{X} \subseteq \mathbb{C P}^{n}$ be a smooth projective (algebraic and compact) variety. Consider on $\mathcal{X}$ the induced Zariski topology (cf. [8] Ch. 1). Let $\mathcal{Y}$ be a Zariski open set of $\mathcal{X}, \mathcal{Y}$ is a quasi-projective variety. Let $E$ be a rank $e$ an algebraic vector bundle over $\mathcal{Y}$. A section $s$ of $E$ defines a map $s: \mathcal{O}_{\mathcal{Y}} \rightarrow E$ and its dual $s^{*}: E^{*} \rightarrow \mathcal{O}_{\mathcal{Y}}$. Here $\mathcal{O}_{\mathcal{Y}}$ is the sheaf of the regular algebraic functions on $\mathcal{Y}$ : If $U$ is an open set of $\mathcal{Y}, \Gamma\left(U, \mathcal{O}_{\mathcal{Y}}\right)$ is the space of the holomorphic functions on $U$ obtained by restriction of rational functions of $\mathbb{C P} \mathbb{P}^{n}$. The image $\mathcal{J}$ of $s^{*}$ is an ideal sheaf. The scheme structure on $\mathcal{Z}=\{z \in Y: s(z)=0\}$ is given by the sheaf $\mathcal{O}_{\mathcal{Z}}=\mathcal{O}_{\mathcal{Y}} / \mathcal{J}$. We say that $\mathcal{Z}$ has the expected dimension if $\operatorname{dim}(\mathcal{Z})=\operatorname{dim}(\mathcal{Y})-e$. Let $T_{\mathcal{Y}, z}$ be the holomorphic tangent space of $\mathcal{Y}$ at $z$ and $d s: T_{\mathcal{Y}, z} \rightarrow E_{z}$ be (induced by) the differential of $s$. If $z \in \mathcal{Z}, \operatorname{ker}(d s) \cong\left(\mathcal{J}_{z} /\left(\mathcal{J}_{z}\right)^{2}\right)^{*}$ is the Zariski tangent space (see [8] §II Ex. 2.8) of $\mathcal{Z}$ at $z$. We say that $z$ is smooth if $d s$ is surjective. By implicit function theorem the smooth points define a codimension $e$ subvariety $\mathcal{Z}^{\prime}$ of $\mathcal{Z}, \operatorname{ker}(d s)$ is the holomorphic tangent space of $\mathcal{Z}^{\prime}$ at $z$.

(2.7). A closed algebraic subset of $\mathcal{Z}$ is called a component if it contains some open subset of $\mathcal{Z}$. We are ignoring here the immersed components (cf. 
[8] Ch. $2 \S 3)$. Clearly $\mathcal{Z}$ is union of its components. A component of $\mathcal{Z}$ is good (reduced) if it contains a smooth point. An algebraic set $\mathcal{Z}$ is reduced if all its components are good and irreducible if $\mathcal{Z}$ is itself a component. Then any smooth point defines a good component of $\mathcal{Z}$. Therefore the closure of a connected component (in the usual topological sense) of $\mathcal{Z}^{\prime}$ is a component (in the algebraic sense) of $\mathcal{Z}$ which is good by definition. Note that a good component has the expected dimension.

(2.8) Examples. The algebraic smoothness is more than the usual one. Set $\mathcal{Y}=\mathbb{C P}^{2}, E=\mathcal{O}_{\mathbb{C P}^{2}}(2)(e=1)$. The coordinate $z_{i}$ gives as in $(0.5)$ a section of $\mathcal{O}_{\mathbb{C P}^{2}}(1)$, then the homogeneous forms of degree 2 define sections of $E$ :

a) $s=\sum_{i=1}^{3} z_{i}^{2} ; \mathcal{Z}=\mathcal{Z}^{\prime}, Q=\left\{\left[z_{1}, z_{2}, z_{3}\right] \in \mathbb{C P}^{2}: \sum_{i=1}^{3} z_{i}^{2}=0\right\}, Q$ is a good component;

b) $s=z_{1} z_{2}: \mathcal{Z}=L_{1} \cup L_{2}, L_{i}=\left\{\left[0, z_{2}, z_{3}\right] \in \mathbb{C P}^{2}: z_{i}=0\right\} ; \mathcal{Z}^{\prime}=\mathcal{Z}-[(0,0,1)]$ : $L_{1}$ and $L_{2}$ are good components, $\mathcal{Z}$ is reduced but not irreducible;

c) $s=z_{1}^{2} ; \mathcal{Z}=L_{1}, \mathcal{Z}^{\prime}=\varnothing: \mathcal{Z}$ is irreducible and not reduced, no good components.

(2.9). Recall that $\operatorname{dim}(\mathcal{P}(F))=\operatorname{dim}(\mathcal{H}(F))+1$ and $\operatorname{dim}(\mathcal{F}(F))=$ $\operatorname{dim}(\mathcal{M}(F))+4$. The computations (2.1) give that any component of our spaces has dimension bigger or equal than the expected one. Then (cf. 2.6) $\mathcal{H}, \mathcal{H}(F), \mathcal{M}, \ldots$ have the expected dimension if the inequalities in (2.1)...(2.5) are equalities. The expected dimension of $\mathcal{F}$ is $2(d-k)-3(2 g+$ $n-1)$ the one of $\mathcal{F}(p)$ is $2(d-k)-3(2 g+n-1)-2: \mathcal{G}=\cup_{p \in X} \mathcal{F}(p)$ should be a divisor in $\mathcal{F}$.

(2.10) Example. We present a case where the inequality (2.1) is sharp. Let $p=Z$ be a point, $H=H^{1}(X, \mathbb{C})$ and $L$ be a spin structure of $X$ : $L^{2}=\omega_{X}$. Take $D=(2 g+1) p, s \in H^{0}(X, L(2 g+1) p)$ half-exact and $F \in$ $H^{0}\left(X, \mathcal{O}_{X}(4 g+1) p\right)$ such that $d F=s^{2}$. The ramification of $F$ contains the zero divisor $E$ of $s$. Note that $\operatorname{deg}(E)=3 g$ and that expected dimension of $\mathcal{H}$ is zero. Varying the moduli of $X$ and $p$ we find a variety of dimension $\geq 3 g-2$.

Let $\Gamma$ be the Hurwitz scheme of the degree $2 g+1$ coverings of $\mathbb{C P}^{1}=\mathbb{C} \cup \infty$ ramified on $3 g+1$ points as follows: Total ramification on $\infty$ and 3 to 1 on the other points. By Hurwitz' formula the curves have genus $g$ and $\operatorname{dim}(\Gamma)=3 g-2$, i.e. the coverings coming from half-exact differentials fill up a component of $\Gamma$. It implies that if $X$ and $p$ are general the expected dimension is the actual one. An easy analysis shows that the general points are smooth. Then (2.2) gives that there are $2^{2 g}$ of such coverings. 


\section{Infinitesimal computation.}

We study the Zariski tangent (see 2.6) of the spaces we have defined in (cf. 1.1 and 1.4). Take a holomorphic curve $s(t)$ in $\mathcal{P}, s(0)=s$. There is a curve $G(t)$ in $R$ such that

$$
s(t)^{2}=d G(t)
$$

Expanding we get

$$
s^{2}+2 s s^{\prime}+o\left(t^{2}\right)=d G(0)+t d G^{\prime}+o\left(t^{2}\right),
$$

where $s^{\prime}=s^{\prime}(0)$ belongs to $V=H^{0}(X, L(D))$ and $F^{\prime}$ to $R=H^{0}\left(X, \mathcal{O}_{X}(2 D-\right.$ $\left.Z^{\prime \prime}\right)$ ). It follows that $s s^{\prime}=\frac{1}{2} d G^{\prime}$ is an exact differential. Denote by $s: V \rightarrow W$ the multiplication $s(t)=s t$ and define $c(s): V \rightarrow H$ by:

$$
c(s)(t)=\text { De Rham class of }\{s t\} .
$$

The Zariski tangent space $T_{s, \mathcal{P}}$ (cf. 2.6) of $\mathcal{P}$ at $s$ is $C_{s}=\operatorname{ker}(c(s))$. If $c(s)$ is surjective we obtain

$$
\operatorname{dim}\left(T_{s, \mathcal{P}}\right)=\operatorname{dim}\left(C_{s}\right)=\operatorname{dim}(V)-\operatorname{dim}(H) \leq \operatorname{dim} \mathcal{H}+1=\operatorname{dim} \mathcal{P} .
$$

Since always $\operatorname{dim}(\mathcal{P}) \geq \operatorname{dim}\left(T_{s, \mathcal{P}}\right), \operatorname{dim}(\mathcal{P})=\operatorname{dim}\left(T_{s, \mathcal{P}}\right)$. Then $(s)$ is a smooth point and defines a good component (see 2.7) of $\mathcal{P}$. In the same way $C_{s} / \operatorname{span}(s)$ is the Zariski tangent of $\mathcal{H}$ as defined by (2.2). We have seen:

Proposition 3.2. If $c(s)$ is surjective then (s) is a smooth point of a good component $\mathcal{C}$ of $\mathcal{H}: \operatorname{dim}(\mathcal{C})=d+k-2 g-n$.

For any effective divisor $F$ we set

$$
C_{s}(-F)=\operatorname{ker}(c(s): V(-F) \rightarrow H)=V(-F) \cap C_{s} .
$$

If furthermore $s \in V(-F)$ it follows that $C_{s}(-F)$ is the Zariski tangent space to $\mathcal{P}(F)$ at $s$. If the map in (3.3) is surjective s is a smooth point of $\mathcal{P}(F)$. We have the following:

Lemma 3.4. If for $s \in \mathcal{P}(q) c(s): V(-q) \rightarrow H$ is surjective, then there is a holomorphic curve $s(t)$ in $\mathcal{P}, s(0)=s$ and $s^{\prime}(0)(q) \neq 0$, hence $s(t) \in \mathcal{P}(q)$ for small $t \neq 0$.

Proof. The map $c(s): V \rightarrow H$ is also surjective and $\operatorname{dim} C_{s}(-q)=\operatorname{dim} C_{s}-1$. Hence there is $s^{\prime} \in C_{s}-C_{s}(-q)$ and a curve $s(t)$ in $\mathcal{P}, s(0)=s$ and $s^{\prime}(0)=$ $s^{\prime}$. 
(3.5). To study the tangent space of $\mathcal{F}$ and of $\mathcal{M}$ we fix a minimal plane $\Pi$ and a basis $\left(s_{1}, s_{2}\right)$ of $\Pi$. Set $x=\left(s_{1}, s_{2}\right) \in \mathcal{F}$ and let

$$
\gamma(t)=\left(s_{1, t}, s_{2, t}\right)
$$

be a holomorphic curve in $\mathcal{F}$ such that $\gamma(0)=x$. The condition $\gamma(t) \in \mathcal{F}$ implies the existence of rational functions $F_{i, t}, i=1,2$ and 3 , such that

$$
d F_{1, t}=s_{1, t}^{2} ; \quad d F_{2, t}=s_{1, t} s_{2, t} ; \quad d F_{3, t}=s_{2, t}^{2}
$$

First order expansion gives: $s_{i, t}=s_{i}+t v_{i}+O\left(t^{2}\right), i=1,2$, and $F_{j, t}=$ $F_{j}+t G_{j}+o\left(t^{2}\right), j=1,2,3$, and then

$$
2 s_{1} v_{1}=d G_{1}, s_{1} v_{2}+s_{2} v_{1}=d G_{2}, 2 s_{2} v_{2}=d G_{3} .
$$

(3.6). Letting $c_{i}=c\left(s_{i}\right): V \rightarrow H$ and $C_{i}=\operatorname{Ker}\left(c_{i}\right)$ we define $\kappa=\kappa_{s_{1}, s_{2}}$ : $V^{2} \rightarrow H^{3}$ by:

$$
\begin{aligned}
\kappa\left(v_{1}, v_{2}\right) & =\left(c_{1}\left(v_{1}\right), c_{1}\left(v_{2}\right)+c_{2}\left(v_{1}\right), c_{2}\left(v_{1}\right)\right) \\
& =\text { de Rham classes of }\left\{\left(2 s_{1} v_{1}, s_{1} v_{2}+s_{2} v_{1}, 2 s_{2} v_{2}\right)\right\} .
\end{aligned}
$$

The tangent space to $\mathcal{F}$ at $x$ is $C_{s_{1}, s_{2}}=\operatorname{ker}(\kappa)$. If $\kappa$ is surjective then $\operatorname{dim}\left(C_{s_{1}, s_{2}}\right)=2(d+k)-3(g+n-1)$ which is the expected dimension of $\mathrm{f}$ (cf. 2.7). Note that if $\left(v_{1}, v_{2}\right) \in C_{s_{1}, s_{2}}$ then $v_{i} \in C_{i}, i=1,2$.

Proposition 3.7. Let $x=\left(s_{1}, s_{2}\right) \in \mathcal{F}$. If $\kappa: V^{2} \rightarrow H^{3}$ is surjective then $x$ is a smooth point of $\mathcal{F}$.

(3.8). If $\kappa$ is surjective then $c_{i} i=1,2$, are surjective, i.e. $s_{1}$ and $s_{2}$ are smooth points of $\mathcal{P}$. Define $\chi_{s_{1}, s_{2}}=\chi: C_{1} \oplus C_{2} \rightarrow H$ by

$$
\begin{aligned}
\chi\left(v_{1}, v_{2}\right)=c_{1}\left(v_{2}\right)+c_{2}\left(v_{1}\right)=\text { De Rham class of }\left\{s_{1} v_{2}+s_{2} v_{1}\right\} \\
s_{i} v_{i}=d G_{i} i=1,2 .
\end{aligned}
$$

Set $f=\frac{s_{2}}{s_{1}}$ we get $s_{1} v_{2}+s_{2} v_{1}=f^{-1} d G_{1}+f d G_{2}$, and hence:

$$
\begin{aligned}
\chi\left(v_{1}, v_{2}\right) & =\text { De Rham class of }\left\{f^{-1} d G_{1}+f d G_{2}\right\} \\
& =\text { De Rham class of }\left\{-\left(f^{-2} G_{1}-G_{2}\right) d f\right\} .
\end{aligned}
$$

The surjectivity of $\chi$ means exactly that the elements of the type $(0, h, 0)$, where $h$ is in $H$, are in the image of $\kappa$. We obtain:

$\kappa$ is surjective $\Leftrightarrow \chi, c_{1}$ and $c_{2}$ are surjective. 
(3.9). We shortly turn to the Zariski tangent space of $\mathcal{F}\left(s_{1}\right)$ at $x=\left(s_{1}, s_{2}\right)$. This can be identified with $K_{s_{1}, s_{2}}$ the kernel of $\rho=\rho_{s_{1}, s_{2}}: V \rightarrow H^{2}$ :

$$
\rho(v)=\left(c_{1}(v), c_{2}(v)\right)=\text { De Rham class of }\left\{s_{1} v, s_{2} v\right\} .
$$

The inclusion $\mathcal{F}\left(s_{1}\right) \subset \mathcal{F}$ gives the injection $K_{s_{1}, s_{2}} \rightarrow C_{s_{1}, s_{2}}:(v) \rightarrow(0, v)$. If $\rho$ is onto $x$ is a smooth point (cf. 2.8) of $\mathcal{F}\left(s_{1}\right)$. If $v \in C_{1}$ then $s_{1} v=d G$, thus:

$$
\begin{aligned}
c_{2}(v) & =\text { De Rham class of }\left\{s_{2} v\right\} \\
& =\text { De Rham class of }\{f d G\}=\text { De Rham class of }\{-G d f\} .
\end{aligned}
$$

We have:

$$
c_{2}: C_{1} \rightarrow H \text { and } c_{1}: C_{2} \rightarrow H \text { are onto } \Leftrightarrow \rho \text { is onto } \Rightarrow \kappa \text { is onto. }
$$

(3.11). If $F$ is an effective divisor $x=\left(s_{1}, s_{2}\right) \in \mathcal{F}(F)$. The tangent spaces of $\mathcal{F}(F)$ and $\mathcal{F}\left(s_{1}, F\right)$ at $x$ are $C_{s_{1}, s_{2}}(-F)=\operatorname{ker}\left(\kappa: V^{2}(-F) \rightarrow H^{3}\right)$ and $K_{s_{1}, s_{2}}(-F)=\operatorname{ker}\left(\rho: V(-F) \rightarrow H^{2}\right)$ respectively. If the above maps are surjective the corresponding components are good. In particular if $\kappa: V^{2}(-p) \rightarrow H^{3}$ is surjective for any $p \in X$ then there is component $\mathcal{K}$ of $\mathcal{F}$ such that $\operatorname{dim}(\mathcal{K})>\operatorname{dim}\left(\mathcal{K} \cap\left(\cup_{p \in X} \mathcal{F}(p)\right)\right)$ and hence $\mathcal{F} \neq \cup_{p \in X} \mathcal{F}(p)$. This will be our strategy in proving the existence of immersed minimal surfaces.

\section{Technical lemmas.}

We need to prove a simple result about the ramification of rational functions on $X$. Let $A=\sum_{i=1}^{a} a_{i} q_{i}$ and $B=\sum_{i=1}^{b} b_{i} r_{i}$ be two effective divisors with disjoint supports: $[A] \cap[B]=\varnothing,[A]=\sum_{i=1}^{a} q_{i}$ and $[B]=\sum_{i=1}^{b} r_{i}, \operatorname{deg}([A])=$ $a$ and $\operatorname{deg}([B])=b$. The problem is to know if the De Rham map

$$
c: H^{0}\left(X, \omega_{X}(A-B)\right) \rightarrow H^{1}(X-[A], \mathbb{C})
$$

is surjective. Set $A^{\prime}=A-[A]$ and write $A=A^{\prime}+\left[A^{\prime}\right]+A^{\prime \prime}$. If $a_{i}>1$ for $i \leq a^{\prime}$ then $A^{\prime}=\sum_{i=1}^{a^{\prime}}\left(a_{i}-1\right) q_{i}$ and $A^{\prime \prime}=\sum_{i=a^{\prime}+1}^{a} q_{i}$.

Let $\mathcal{R}=\mathcal{R}(A, B)$ be the sheaf of the rational functions on $X$ with poles at $A^{\prime}$ and ramified at $B$. If $U$ is an open set of $X$ :

$$
\Gamma(U, \mathcal{R}(A, B))=\left\{f: f \in \Gamma\left(U, \mathcal{O}_{X}(A-[A])\right) \text { and } d f \in \Gamma\left(U, \omega_{X}(-B)\right)\right\} .
$$

Now $\mathcal{O}_{X}\left(A^{\prime}-B-[B]\right)$ is a subsheaf of $\mathcal{R}$ and there is a sheaf exact sequence 
$(4.1)$.

$$
0 \rightarrow \mathcal{O}_{X}\left(A^{\prime}-B-[B]\right) \rightarrow \mathcal{R} \rightarrow \mathcal{O}_{[B]} \rightarrow 0,
$$

where $\mathcal{R} \rightarrow \mathcal{O}_{[B]}$ is the evaluation at $[B]$. Exterior differential gives another exact sequence:

$(4.2)$.

$$
0 \rightarrow \mathbb{C} \rightarrow \mathcal{R} \stackrel{d}{\rightarrow} \omega_{X}\left(A^{\prime}+\left[A^{\prime}\right]-B\right) \subset \omega_{X}(A-B) .
$$

Taking residues we obtain a surjection $\omega_{X}(A-B) \stackrel{\text { res }}{\longrightarrow} \mathcal{O}_{[A]}$. Its kernel, $\omega_{X}(A-B)^{0}$, which is also the kernel of $\omega_{X}\left(A^{\prime}-B\right) \stackrel{\text { res }}{\longrightarrow} \mathcal{O}_{\left[A^{\prime}\right]}$, is the image of $d, d(\mathcal{R})=\omega_{X}(A-B)^{0}$. Hence (4.2) defines two short exact sequences:

$$
\begin{gathered}
0 \rightarrow \mathbb{C} \rightarrow \mathcal{R} \stackrel{d}{\rightarrow} \omega_{X}(A-B)^{0} \rightarrow 0 \\
0 \rightarrow \omega_{X}(A-B)^{0} \rightarrow \omega_{X}(A-B) \stackrel{\text { res }}{\longrightarrow} \mathcal{O}_{[A]} \rightarrow 0 .
\end{gathered}
$$

Therefore the vanishing of $H^{1}(X, \mathcal{R})$ gives that the coboundary maps

$H^{0}\left(X, \omega_{X}(A-B)^{0}\right) \rightarrow H^{1}(X, \mathbb{C})$ and $H^{1}\left(X, \omega_{X}(A-B)^{0}\right) \rightarrow H^{2}(X, \mathbb{C})=\mathbb{C}$

are both surjective and the second one is an isomorphism. The image of

$$
H^{0}\left(X, \omega_{X}(A-B)\right) \stackrel{\text { res }}{\longrightarrow} H^{0}\left(X, \mathcal{O}_{[A]}\right)=\mathbb{C}^{a}
$$

is the hyperplane $\mathcal{L}=\left\{\sum_{i=1}^{a} x_{i}=0\right\}$ by the residues theorem, this implies $H^{1}\left(X, \omega_{X}(A-B)\right)=0$ and that the maps $H^{0}\left(X, \omega_{X}(A-B)^{0}\right) \rightarrow$ $H^{1}(X, \mathbb{C})$ and $H^{0}\left(X, \omega_{X}(A-B)\right) \rightarrow \mathcal{L}$ are both surjective. This show that $c: H^{0}\left(X, \omega_{X}(A-B)\right) \rightarrow H^{1}(X-[A], \mathbb{C})$ is also a surjection.

Conversely if $c$ is onto then $H^{0}\left(X, \omega_{X}(A-B)^{0}\right) \rightarrow H^{1}(X, \mathbb{C})$ is surjective and $H^{1}\left(X, \omega_{X}(A-B)\right)=0(\operatorname{deg}(A-B) \geq g+1>0)$. This forces $H^{1}\left(X, \omega_{X}(A-B)^{0}\right)=\mathbb{C}$ and therefore $H^{1}(X, \mathcal{R})=0$. Summarizing we have:

Proposition 4.4. The map $c: H^{0}\left(X, \omega_{X}(A-B)\right) \rightarrow H^{1}(X-[A], \mathbb{C})$ is onto if and only if $H^{1}(X, \mathcal{R}(A, B))=0$.

From $(4.1) H^{1}\left(X, \mathcal{O}_{X}\left(A^{\prime}-B-[B]\right)\right)=0$ implies $H^{1}(X, \mathcal{R})=0$, we get:

Lemma 4.5. If $\operatorname{deg}(A-B)-a-b>2 g-2$ then $H^{1}(X, \mathcal{R}(A, B))=0$.

We will give some applications. Let $M$ be a line bundle on $X$ and $\sigma \in$ $H^{0}(X, M)$ be a section of $M$. By composing c with the multiplication $\sigma$ : $H^{0}\left(X, \omega_{X}(A-B) \otimes M^{-1}\right) \rightarrow H^{0}\left(X, \omega_{X}(A-B)\right)$, we obtain (compare with $3.1)$ : 
(4.6).

$$
c(\sigma): H^{0}\left(X, \omega_{X}(A-B) \otimes M^{-1}\right) \rightarrow H^{1}(X-[A], \mathbb{C}) .
$$

Let $E$ be the zero divisor of $\sigma, M$ is isomorphic to $\mathcal{O}_{X}(E)$ and s defines the exact sequence:

$(4.7)$.

$$
0 \rightarrow \omega_{X}(A-B) \otimes M^{-1} \stackrel{\sigma}{\rightarrow} \omega_{X}(A-B) \rightarrow \omega_{X}(A)_{\mid E} \rightarrow 0,
$$

then the image of $\sigma: H^{0}\left(X, \omega_{X}(A-B) \otimes M^{-1}\right) \rightarrow H^{0}\left(X, \omega_{X}(A-B)\right)$ is $H^{0}\left(X, \omega_{X}(A-B-E)\right)$. It follows that $c(\sigma)$ is onto if and only if $H^{0}\left(X, \omega_{X}(A-\right.$ $B-E)) \rightarrow H^{1}(X-[A], \mathbb{C})$ is surjective. Write $E=E^{\prime}+E^{\prime \prime},\left[E^{\prime}\right] \subset[A]$ and $E^{\prime \prime} \cap A=\varnothing$. If $A-E^{\prime}$ is effective and $\left[A-E^{\prime}\right]=[A]$. Applying (4.5) and (4.6) to $A-E^{\prime}$ and $E^{\prime \prime}+B$ we obtain:

Lemma 4.8. If $\operatorname{deg}(A-B-[A+B+E])>2 g-2+\operatorname{deg}(M)$ and $[A]=[A-E \cap A]$ then $c(\sigma)$ is surjective.

Let $\mathbb{P}$ be the projective space of $H^{0}(X, M)$ and $U$ be a compact algebraic subset of $\mathbb{P}$. Put $\operatorname{dim}(U)=u$ and define the Zariski open set of $U$ :

$$
U^{\prime}=\{(\sigma) \in U: c(\sigma) \text { is surjective }\} .
$$

We fix a point $q$ of $X, q \notin[A], A=\sum_{i=1}^{a} a_{i} q_{i}$, and we find $(\sigma) \in U$ with zero divisor $E=u q+\Delta, \Delta$ effective and $\operatorname{deg}([\Delta]) \leq \operatorname{deg}(M)-u$. If $a_{i}>$ $\operatorname{deg}(M)-u$ then the multiplicity condition is satisfied. This gives:

Lemma 4.9. If $u \geq \max \left(\operatorname{deg}(M)-a_{i}+1,2 g-2+a+b+\operatorname{deg}(2 M+B-A)\right)$ there is $(\sigma) \in U$ such that $c(\sigma)$ is surjective, i.e. $U^{\prime}$ is non empty.

(4.10). To apply the previous results to minimal surfaces we need to consider the slightly more sophisticated (3.6), (3.8) and (3.10) situations. Let $f$ be a non constant rational function with polar divisor $F$. It follows $f \in H^{0}\left(X, \mathcal{O}_{X}(F)\right)$ and $d f \in H^{0}\left(X, \omega_{X}(F+[F])\right)$. Let $A$ and $B$ be as before and $\Omega$ be the zero divisor of $d f$. As in (4.7) we have an exact sequence:

$$
0 \rightarrow \mathcal{O}_{X}(A-B) \stackrel{d f}{\longrightarrow} \omega_{X}(A+F+[F]-B) \rightarrow \omega_{X}(A+F+[F]-B)_{\Omega} \rightarrow 0 .
$$

Set $F^{\prime \prime}=(F+[F]) \cap B, B^{\prime}=B-F^{\prime \prime}$ and $F^{\prime}=F+[F]-F^{\prime \prime}$. We compose c with the multiplication: $d f: H^{0}\left(X, \mathcal{O}_{X}(A-B)\right) \rightarrow H^{0}\left(X, \omega_{X}\left(A+F^{\prime}-B^{\prime}\right)\right)$ and obtain:

$$
c(d f): H^{0}\left(X, \mathcal{O}_{X}(A-B)\right) \rightarrow H^{1}\left(X-\left[A+F^{\prime}\right], \mathbb{C}\right) .
$$


If $G \in H^{0}\left(X, \mathcal{O}_{X}(A-B)\right)$ then:

$$
c(d f)(G)=\text { De Rham class of }\{G d f\} .
$$

We apply (4.8) where $M=\omega_{X}(F+[F])$ and $\sigma=d f$. The roles of the $A$ and $B$ are taken by $A+F^{\prime}$ by $B^{\prime}=B-F^{\prime \prime}$. To use (4.8) we need:

$$
\operatorname{deg}(A-B)+\operatorname{deg}(F)>2(g-1)+\operatorname{deg}(W)+\operatorname{deg}([A+F+W+B]) .
$$

Since $\operatorname{deg}(W)=2 g-2+\operatorname{deg}(F+[F])$ it gives $\operatorname{deg}(A-B)>4(g-1)+$ $\operatorname{deg}([F])+\operatorname{deg}[A+F+\Omega+B] \geq 4(g-1)+2 \operatorname{deg}(F)+\operatorname{deg}(\Omega)+\operatorname{deg}([A+B]) \geq$ $6(g-1)+4 \operatorname{deg}(F)+a+b$.

Since the multiplicity of the point of $\Omega$ is less than $\operatorname{deg}(f)$ and $\Omega$ is disjoint from $[F]$ the other inequalities are $a_{i} \geq \operatorname{deg}(f)$. Hence:

Lemma 4.11. If $a_{i} \geq \operatorname{deg}(f)$ for any $i$ and $\operatorname{deg}(A-B-[B+A])>$ $6 g-6+4 \operatorname{deg}(f)$ then $c(d f): H^{0}\left(X, \mathcal{O}_{X}(A-B)\right) \rightarrow H^{1}\left(X-\left[A+F^{\prime}\right], \mathbb{C}\right)$ is surjective.

We need a "dual" version of the above lemma, we assume

$$
a_{i}>\operatorname{deg}(f) \text { for all } i \text {. }
$$

Let $\mathcal{R}=\mathcal{R}(A, B)$ and $d(\mathcal{R})$ be defined as in (4.3). Multiplication by $f$ gives $0 \rightarrow \omega_{X}(A-B) \stackrel{. f}{\longrightarrow} \omega_{X}(A+F-B)$. Write $F=F^{\prime}+F^{\prime \prime}, F^{\prime \prime}=B \cap F$ and $B^{\prime}=B-F^{\prime \prime}$. Set $T=\left[A+F^{\prime}\right]$ and define $c(f): H^{0}(X, \mathcal{R}) \rightarrow H^{1}(X-T, \mathbb{C})$ by:

$$
c(f)(G)=\text { De Rham class of }\{f d G\} .
$$

From the inclusion: (4.1) $H^{0}\left(X, \mathcal{O}_{X}(A-[A]-B-[B])\right) \rightarrow H^{0}(X, \mathcal{R})$ we have

$$
c^{\prime}(f): H^{0}\left(X, \mathcal{O}_{X}(A-[A]-B-[B])\right) \rightarrow H^{1}(X-T, \mathbb{C}) .
$$

Now Leibnitz rule, $d(f G)=G d f+f d G$ says that the De Rham classes of $f d G$ and of $-G d f$ are the same: $c^{\prime}(f)=-c(d f)$. The following implication holds:

$c(d f)$ is surjective $\Rightarrow c^{\prime}(f)$ is surjective $\Rightarrow c(f)$ is surjective.

Since, from $(*) T=\left[A-[A]+F^{\prime}\right]$ we rewrite (4.11) for $A^{\prime}=A-[A]$ and $B^{\prime}=B+[B]$ :

Lemma 4.12. If $a_{i}>\operatorname{deg}(f)$ and $\operatorname{deg}(A-B)-2(\operatorname{deg}[A]+[B])>(g-1)+$ $4 \operatorname{deg}(f)$ for all $i$, then $c(f): H^{0}(X, \mathcal{R}) \rightarrow H^{1}\left(X-\left[A+F^{\prime}\right], \mathbb{C}\right)$ is surjective.

(4.13). Take two independent global sections of $M$, say $\sigma_{1}$ and $\sigma_{2}$. Let $E_{i}$, $i=1,2$, be their zero divisors and $c_{i}=c\left(\sigma_{i}\right): H^{0}\left(X, \omega_{X}(A-B) \otimes M^{-1}\right) \rightarrow$ 
$H^{1}(X-[A], \mathbb{C})$ be as in (4.6). Set $C_{i}=\operatorname{ker}\left(c_{i}\right)$. By restriction we define $c_{i}: C_{j} \rightarrow H^{1}(X-[A], \mathbb{C}), i \neq j$, and $\chi\left(\sigma_{1}, \sigma_{2}\right): C_{1} \oplus C_{2} \rightarrow H^{1}(X-[A], \mathbb{C})$ (compare with 3.8) by

$$
\chi\left(\sigma_{1}, \sigma_{2}\right)(x, y)=c_{2}(y)+c_{1}(x) .
$$

Set $f=\sigma_{1} / \sigma_{2}$. If $y \in C_{1}$, then $\sigma_{1} y=d G$ and hence $\sigma_{2} y$ can be written

$$
\sigma_{2} y=f\left(\sigma_{1} y\right)=f d G .
$$

The polar and zero divisors of $f$ are respectively $F_{2}=E_{2}-E_{1} \cap E_{2}$ and $F_{1}=E_{1}-E_{1} \cap E_{2}$. We write $E_{1}=E^{\prime}+E^{\prime \prime}$ where $\left[E^{\prime}\right] \subset[A]$ and $\left[E^{\prime \prime}\right] \cap[A]=\varnothing$. Set $\Delta=B+E^{\prime \prime}$.

(**) We assume $a_{i}>\operatorname{deg}(f)+\operatorname{deg}\left(E^{\prime}\right)+1$ for all $i$.

By $(* *) C=A-E^{\prime}=\sum_{i=1}^{a} c_{i} q_{i}$ and $c_{i}>1, C-[A]$ is effective and $[A]=[C-[A]]$. The rational function $G$ of $(4.14)$ belongs to $H^{0}(X, \mathcal{R}(C, \Delta))$. As we have observed in the proof of (4.8) the image of the multiplication

$$
\sigma_{i}: H^{0}\left(X, \omega_{X}(A-B) \otimes M^{-1}\right) \rightarrow H^{0}\left(X, \omega_{X}(A-B)\right)
$$

is $H^{0}\left(X, \omega_{X}\left(A-B-E_{i}\right)\right), i=1,2$. In particular $d G \in H^{0}\left(X, \omega_{X}(A-B-\right.$ $\left.\left.E_{1}\right)\right) \Leftrightarrow f d G \in H^{0}\left(X, \omega_{X}\left(A-B-E_{2}\right)\right)=\operatorname{Im}\left(\sigma_{2}\right) \Leftrightarrow \exists z \in H^{0}\left(X, \omega_{X}(A-\right.$ $\left.B) \otimes M^{-1}\right)$ and $\sigma_{2} z=f\left(\sigma_{1} z\right)=f d G$.

We have $\sigma_{1} z=d G$, that is $z \in C_{1}$. Hence the following implication holds:

$$
\begin{aligned}
c_{2}: C_{1} \rightarrow H^{1}(X-[A], \mathbb{C}) \text { is onto } \Leftrightarrow c(f): & H^{0}(X, \mathcal{R}(C, \Delta)) \\
& \rightarrow H^{1}(X-[A], \mathbb{C}) \text { is onto. }
\end{aligned}
$$

We apply (4.12) where $C$ and $\Delta$ take the place of $A$ and of $B$. Note that in our case $[T]=[C]=[A]$. The inequalities in (4.12) are the $(* *)$ and

(***) $\quad \operatorname{deg}(C-\Delta)-2 \operatorname{deg}([C]+[\Delta])>6(g-1)+4 \operatorname{deg}(f)$.

Now we have: $\operatorname{deg}(f)+\operatorname{deg}\left(E_{1} \cap E_{2}\right)=\operatorname{deg}(M)=\operatorname{deg}\left(E_{1}\right) C=A-E^{\prime}$,

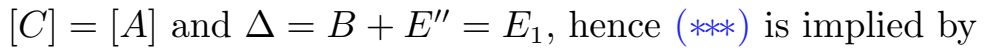

$$
\begin{aligned}
\operatorname{deg}(A-B)-2 \operatorname{deg}([A])-2 \operatorname{deg}([B])-2 \operatorname{deg}\left[E^{\prime \prime}\right] & \\
& >6(g-1)+5 \operatorname{deg}(M)-4 \operatorname{deg}\left(E_{1} \cap E_{2}\right) .
\end{aligned}
$$

Finally using that $\left[E^{\prime \prime}\right]=\left[E_{1}\right]-[A]$ we have:

Lemma 4.15. If $a_{i}>\operatorname{deg}\left(M-E_{1} \cap E_{2}\right)+\operatorname{deg}\left(E^{\prime}\right)+1$ for any $i$ and $\operatorname{deg}(A-B)-4 a-2 b>6(g-1)+2 \operatorname{deg}\left(\left[E_{1}\right]\right)+5 \operatorname{deg}(M)-4 \operatorname{deg}\left(E_{1} \cap E_{2}\right)$ then $c_{2}$ is surjective. A "fortiori" $\chi: C_{1} \oplus C_{2} \rightarrow H^{1}(X-[A], \mathbb{C})$ is surjective. 


\section{Proof of the main result.}

We apply the results of $\S 4$ to our situation: $M=L(D), D=\sum_{i=1}^{n} n_{i} p_{i}$, $[D]=Z, A=2 D+Z^{\prime}, B=\varnothing, \omega_{X}(A) \otimes M^{-1}=L(D), V=H^{0}(X, L(D))$ and $H=H^{1}(X-Z, \mathbb{C})$.

Proposition 5.1. Assume: i) $2 n_{i} \geq 3 g-1+n$ if $i \leq 2 k$; ii) $2 n_{i} \geq 3 g+n$ if $i>2 k$; iii) $d+k \geq 6 g+2 n-4$, then any component $\mathcal{C}$ of $\mathcal{H}$ is good.

Proof. Let $\mathcal{C}$ be a component of $\mathcal{H}$. Put $\operatorname{dim}(\mathcal{C})=u=d+k-v$. We know that (cf. 2.1 and 2.8) $v \leq 2 g+n$. The hypotheses of (4.9) need exactly:

i) $v \leq 2 g+n \leq 2 n_{i}-g+1, i \leq 2 k$; ii) $v \leq 2 n_{i}-g$, $i>2 k$; iii) $v \leq 2 g+n \leq$ $d+k-4(g-1)-2 n$.

We find $(s) \in \mathcal{C}$ such that $c(s)$ is surjective, hence (cf. 2.8 and 3.2$) \mathcal{C}$ is a good component.

Set as usual $V(-q)=H^{0}(X, L(D-q))$, and $\mathcal{H}(q)=\mathcal{H} \cap \mathbb{P}(V(-q))$ where the $q$ is a point of $X$. Arguing as before a reasonable statement that avoids the cases $q \in Z, q \in Z^{\prime}$ and $q \in Z^{\prime \prime}$ is the following:

Proposition 5.2. Assume $d+k \geq 6 g+2 n-2$ and $2 n_{i} \geq 3 g+2+n$ for all $i$. Then for any $q \in X, \mathcal{H}(q)$ is a generically smooth divisor of $\mathcal{H}$.

For completeness we prove:

Corollary 5.3. If $2 n_{i} \geq 3 g+n$ and $d+k \geq 8 g+3 n-4 \mathcal{H}$ is irreducible.

Proof. Assume we have two components $\mathcal{C}$ and $\mathcal{D}$ of $\mathcal{H}$. Then $\operatorname{dim}(\mathcal{C} \cap \mathcal{D}) \geq$ $d+k-2(2 g+n)$. Now $\mathcal{C} \cap \mathcal{D}$ is contained in $\mathcal{H}_{\text {sing }}$ the singular locus of $\mathcal{H}$. By $(4.9)$ and $(5.1)$ we get $\operatorname{dim}\left(\mathcal{H}_{\text {sing }}\right)<4(g-1)+n$. This gives

$$
(d+k)-2(2 g+n)<4(g-1)+n .
$$

Hence $d+k \geq 8 g+3 n-4$ which implies that $\mathcal{H}$ is irreducible.

(5.4). We would like to use (4.15) to study minimal surfaces. Writing as usual $\mathcal{M}(F)=\mathcal{M} \cap G(2, V(-F))$ we know from (2.4) that

$$
\operatorname{dim}(\mathcal{M}(F)) \geq 2(d+k)-3(g+n)-1-2 \operatorname{deg}(F) .
$$

Take $F=m q$ where $q \in Z$. To be sure that $\mathcal{M}(m q)$ is not empty we assume:

$$
2(d+k-m) \geq 3(2 g+n)+1
$$


Then there are $\sigma_{1}$ and $\sigma_{2}$ in $V(-m q)$ such that $\Pi=\operatorname{span}\left(\sigma_{1}, \sigma_{2}\right)$ is minimal. Recall (cf. 3.6) and (4.13) the spaces $C_{i}=\operatorname{ker}\left(c\left(\sigma_{i}\right)=V \rightarrow H\right.$ ) and the restrictions $c_{i}: C_{j} \rightarrow H\{i, j\}=\{1,2\}$. Let $E_{i}$ be the zero divisors of $\sigma_{i}$, $i=1,2$, we have: $\operatorname{deg}\left(\left[E_{1}\right]\right) \leq(d+k)+g-m, \operatorname{deg}\left(E_{1} \cap E 2\right) \geq m$. We rewrite $E_{1}=E^{\prime}+E^{\prime \prime}$ where $\left[E^{\prime}\right] \subset[2 D+Z]$ and $\left[E^{\prime \prime}\right] \cap[2 D+Z]=\varnothing: \operatorname{deg}\left(E^{\prime}\right) \leq$ $(d+k)+(g-1)-m$. We first use (4.15) with $A=2 D+Z$ and $B=\varnothing$, this requires:

$$
\begin{gathered}
2 n_{i} \geq 2(d+k-m)+2 g+1 \\
2(d+k)-4 n>6(g-1)+2(d+k+g-m)+5(d+k+g-1)-4 m .
\end{gathered}
$$

The second one becomes: $6 m>5(d+k)+13 g+4 n-11$. Coupling with $(*)$ we have to find integer an $m$ such that:

(***) $\quad 6(d+k)-9(2 g+n)-3 \geq 6 m>5(d+k)+13 g+4 n-11$.

This certainly exists if: $6(d+k)-9(2 g+n)-3>(5(d+k)+13 g+4 n-11)+6$. It gives

$$
d+k>31 g+13 n-2 .
$$

Then we take the biggest value of $m$, say $n$. The upper bound reads $2(d+k)-$ $3(2 g+n)-1 \geq 2 m$, i.e. $\nu \geq d+k-3 g-3 n / 2-1: d+k+g-\nu \leq 4 g+3 n / 2-1$. If $2 n_{i} \geq 8 g+3 n-1$ (**) holds: $2 n_{i} \geq 8 g+3 n-1 \geq 2(d+k+g-n)+1$. We have showed:

Lemma 5.5. If $d+k>31 g+13 n-2$ and $2 n_{i} \geq 8 g+3 n-1$ for all $i$, then there is $\left(\sigma_{1}, \sigma_{2}\right) \in \mathcal{F}$ such that $\rho\left(\sigma_{1}, \sigma_{2}\right)$ and $\kappa\left(\sigma_{1}, \sigma_{2}\right)$ are onto.

Using (5.5), (3.7) and (3.11) we get:

Proposition 5.6. Assume $d+k>31 g+13 n-2$ and $2 n_{i} \geq 8 g+3 n-1$ for all $i$. Then there is a good component of $\mathcal{F}$ and of $\mathcal{F}\left(\sigma_{1}\right)$, where $\sigma_{1} \in V$ is a suitable half exact differential.

Recall (see 1.5) that $\mathcal{F}^{\prime}=\mathcal{F}-\cup_{p \in X} \mathcal{F}(p)$. Consider $M=L(D-p), p \in X$, $\sigma_{i} \in V(-p) i=1,2$, and (see 3.3) $C_{i}(-p)=\operatorname{ker}\left(\sigma_{i}: V(-p) \rightarrow H\right)$. Letting $B=p$ and $A=2 D+Z$ if $p \in Z$, or $A=2 D+Z-p$ and $B=\varnothing$ if $p \in Z$, the previous computation gives:

Lemma 5.7. If $d+k>31 g+13 n+8$ and $2 n_{i}>8 g+3 n$ for all $i$, then there is $x=\left(\sigma_{1}, \sigma_{2}\right) \in \mathcal{F}$ such that $\rho: V(-p) \rightarrow H^{2}$ is onto for all $p$ in $X$.

Proposition 5.8. If $d+k>31 g+13 n+8$ and $2 n_{i}>8 g+3 n$ for any $i$ then there is a smooth point $x=\left(\sigma_{1}, \sigma_{2}\right) \in \mathcal{F}^{\prime}$. (Note that $n \geq 8$ and $2 n_{i} \geq 8 g+3 n+1$ imply $d+k>31 g+13 n+8$.)

Proof. Take $y=\left(\sigma_{1}, \sigma_{2}\right)$ given by (5.7). Let $E$ be the zero divisor of $\sigma_{1}$ and (see 3.9) $\rho_{p}: V(-p) \rightarrow H$ be the restriction of $\rho: V \rightarrow H$. If $K=\operatorname{ker}(\rho)$ 
we have $K(-p)=\operatorname{ker}\left(\rho_{p}\right)$. Recall (cf. 3.11) that $K$ and $K(-p), p \in[E]$, are respectively the tangent spaces of $\mathcal{F}\left(\sigma_{1}\right)$ and of $\mathcal{F}\left(\sigma_{1}, p\right)$, at $y$. Since $\rho_{p}$ is onto $\mathcal{F}\left(\sigma_{1}\right)$ is smooth at $y$ and $\operatorname{dim} K(-p)=\operatorname{dim} K-1$. Take $v \in K-\cup_{p \in[E]} K(-p)$ and a holomorphic curve $\gamma(t)=\left(\sigma_{1}, \sigma_{2, t}\right) \in \mathcal{F}\left(\sigma_{1}\right)$ such that $\gamma(0)=y$, $\gamma^{\prime}(0)=v$. As in (3.4) $\sigma_{2, t}$ does not vanish on $[E]$ for some $t \neq 0$. Fix such a $t, \sigma_{1}=s_{1}$ and $\sigma_{2, t}=s_{2}$. Then $\Pi=\operatorname{span}\left(s_{1}, s_{2}\right)$ is a minimal base point free plane.

Our theorem has been proved. By convenience we repeat some details:

Proof of the Theorem. Fixed $Z$ we can take for instance $L^{2}=\omega_{X}, k=0$ and $D=m Z$. We need $2 m \geq 8 g+3 n+1$ and $n m>31 g+13 n+8$. By (5.7) there is $\left(s_{1}, s_{2}\right) \in \mathcal{F}^{\prime}$. Define as in (1.8) $y=\operatorname{Re} \int \mathcal{W}\left(\left(s_{1}, s_{2}\right)\right)=$ $\operatorname{Re}\left(F_{1}, F_{2}, F_{3}\right)$.

(5.9). In the hypothesis of $(5,8)\left(s_{1}, s_{2}\right)$ defines a good component $\mathcal{G}$ of $\mathcal{F}$, of dimension $2(d-k)-3(2 g+n-1)$. It would be interesting, in analogy with (5.3), to know when $\mathcal{F}=\mathcal{G}$. Varying $X$ and $Z$ as in (1.11) we obtain families of dimension $r=2 d-2 k-3 g-2 n$, provided (cf. 2.7) that $\rho \geq 4$. For $n=1$ we conjecture the existence of non rigid minimal surfaces if $2 d \geq 3 g+6$.

\section{References}

[1] E. Arbarello, M. Cornalba, P. Griffiths and J. Harris, Geometry of algebraic curves, Vol. 1, Grund. Math. Wiss., 267, Berlin, Heidelberg, New York, 1985.

[2] A. Bobenko, Surfaces in terms of 2 by 2 matrices. Old and new integrable cases, in 'Harmonic maps and integrable system' (Ferdy, Wood, eds.), Vieweg, (1994), 82-127.

[3] R. Bryant, A duality theorem for Willmore surfaces, J. Diff. Geom., 20 (1984), 23-53.

[4] E. Calabi, Quelche applications de l'analyse complex aux surfaces d'aire minima, in 'Topics in Complex Manifold' (H. Rossi ed.), Les Presses de l'Univ. de Montreal, 1989.

[5] E. Cartan, The theory of spinors, Dover publ., New York, 1981.

[6] F. Gackstatter and R. Kunert, Konstruction vollständiger Minimalflächen vom endlicher Totalkrümmung, Archive for Rat. Mech. Anal., 65 (1977), 289-297.

[7] P. Griffiths and J. Harris, Principles of algebraic geometry, Wiley Interscience, New York, 1978.

[8] R. Hartshorne, Algebraic geometry, Grad. Text in Math, Springer Verlag Berlin, Heidelberg, New York, 1977.

[9] L. Jorge and W. Meeks III, The topology of complete minimal immersion of finite total Gaussian curvature, Topology, 22 (1983), 201-221.

[10] R. Kusner and N. Schmitt, The spinor representation of Minimal surfaces, preprint. 
[11] H.B. Lawson, Lectures on minimal submanifolds, Publish or Perish Press, Berkeley, 1971.

[12] R. Osserman, Global properties of minimal surfaces in $E^{3}$ and $E^{n}$, Annals of Math. SO(2), 32 (1964), 340-364.

[13] _ A survey of minimal surfaces, New York, Dover pub., 2 Ed., 1986.

[14] U. Pinkall, Regular homotopy classes of immersed surfaces, Topology, 24 (1985), 421-434.

[15] K. Yang, Meromorphic functions on a compact Riemann surface and associated complete minimal surfaces, Proc. of the AMS, 102 (1989), 706-711.

Received April 10, 1996 and revised April 9, 1997. The author was partially supported by $40 \%$ project Geometria Algebrica M.U.R.S.T., by G.N.S.A.G.A. (C.N.R.) Italy and by European project Sci. "Geom. of Alg. variety".

UNIVERSitá Di PAVIA

VIA FERRATA 1

27100 PAVIA, ITALY

E-mail address: pirola@dragon.ian.pv.cnr.it

DIFFERENCES FROM THE PAPER VERSION: WE HAVE CORRECTED A REFERENCE IN 1.3 AND THE TITLE OF SECTION 2. 